HIFAN 1692

\title{
Survey of Collective Instabilities and Beam-Plasma Interactions in Intense Heavy Ion Beams
}

Ronald C. Davidson, Mikhail A. Dorf, Igor D. Kaganovich, Hong Qin, Adam Sefkow and Edward A. Startsev

Princeton Plasma Physics Laboratory, Princeton, New Jersey, USA

Dale R. Welch and David V. Rose

Voss Scientific, Albuquerque, New Mexico, USA

Steven M. Lund

Lawrence Berkeley National Laboratory, Berkeley, California, USA

Accelerator Fusion Research Division

Ernest Orlando Lawrence Berkeley National Laboratory

University of California

Berkeley, California 94720

Nucl. Inst. and Meth. Phys. Res. A, (in press, 2008)

This work was supported by the Director, Office of Science, Office of Fusion Energy Sciences, of the U.S. Department of Energy under Contract No. DE-AC02-05CH11231. 


\title{
Survey of Collective Instabilities and Beam-Plasma Interactions in Intense Heavy Ion Beams
}

\author{
Ronald C. Davidson, Mikhail A. Dorf, Igor D. Kaganovich, Hong Qin, Adam Sefkow and \\ Edward A. Startsev \\ Princeton Plasma Physics Laboratory, Princeton, New Jersey, USA \\ Dale R. Welch and David V. Rose \\ Voss Scientific, Albuquerque, New Mexico, USA \\ Steven M. Lund \\ Lawrence Berkeley National Laboratory, Berkeley, California, USA
}

\begin{abstract}
This paper presents a survey of the present theoretical understanding based on advanced analytical and numerical studies of collective processes and beam-plasma interactions in intense heavy ion beams for applications to ion-beam-driven high energy density physics and heavy ion fusion. The topics include: discussion of the conditions for quiescent beam propagation over long distances; and the electrostatic Harris instability and the transverse electromagnetic Weibel instability in highly anisotropic, intense one-component ion beams. In the longitudinal drift compression and transverse compression regions, collective processes associated with the interaction of the intense ion beam with a charge-neutralizing background plasma are described, including the electrostatic electron-ion two-stream instability, the multispecies electromagnetic Weibel instability, and collective excitations in the presence of a solenoidal magnetic field. The effects of a velocity tilt on reducing two-stream instability growth rates are also discussed. Operating regimes are identified where the possible deleterious effects of collective processes on beam quality are minimized.
\end{abstract}

\section{INTRODUCTION}

This paper presents a survey of the present theoretical understanding based on advanced analytical and numerical studies of collective processes and beam-plasma interactions in intense heavy ion beams for applications to ion-beam-driven high energy density physics and heavy ion 
fusion [1-7]. A complete description of collective processes in intense charged particle beams is provided by the nonlinear Vlasov-Maxwell equations [8-10] for the self-consistent evolution of the beam distribution function, $f_{b}(\mathbf{x}, \mathbf{p}, t)$, and the average electric and magnetic fields $\mathbf{E}(\mathbf{x}, t)$ and $\mathbf{B}(\mathbf{x}, t)$. The effects of finite geometry and intense self-fields often make it difficult to obtain detailed predictions of beam equilibrium, stability and transport properties based on the VlasovMaxwel equations. Nonetheless, often with the aid of advanced numerical simulations, there has been considerable theoretical progress in applying the Vlasov-Maxwell equations to investigate the detailed equilibrium and stability properties of intense charged particle beams [11-80]. These theoretical studies include a wide variety of collective interatction processes ranging from the electrostatic Harris instability [32-42] and electromagnetic Weibel instability [39,43-48] driven by large temperature anisotropy with $T_{\perp b} \gg>T_{\| b}$ in a one-component nonneutral beam, to wallimpedance-driven collective instabilities [49-51], to the resistive hose instability [52-58], to the dipole-mode two-stream (electron cloud) instability for an intense ion beam propagating through a partially neutralizing electron background [59-71], to the collective processes associated with the interaction of the intense ion beam with a charge-neutralizing background plasma [72-77], including the electrostatic electron-ion two-stream instability and the multispecies electromagnetic Weibel instability [78-82], and collective excitations in the presence of a solenoidal magnetic field $[3,80]$, to the development and application of a nonlinear stability theorem in the smooth-focusing approximation $[8,23,24]$.

The present survey of collective processes and beam-plasma interactions affecting intense ion beam propagation is necessarily brief. In the acceleration and beam transport regions, the topics covered in Sec. II include discussion of the sufficient condition for quiescent (stable) beam propagation over long distances; and the electrostatic Harris-type instability and the electromagnetic Weibel-type instability in strongly anisotropic, one-component nonneutral beams. In Sec. III, collective processes associated with the interaction of an intense ion beam pulse with a large-volume charge-neutralizing background plasma are described. To achieve the high focal spot intensities necessary for high energy density physics and heavy ion fusion applications, compressing the beam longitudinally and transversely in the presence of a dense charge-neutralizing background plasma has many attractive features [1-7], particularly because the plasma electrons eliminate (or significantly reduce) the large, defocusing space-charge force of the ion beam pulse. The collective beam-plasma interaction processes summarized in Sec. III 
include: the electrostatic electron-ion two-stream instability; the multispecies electromagnetic Weibel instability; dynamic stabilization of the two-stream instability during longitudinal drift compression; and the effects of solenoidal magnetic field on collective beam-plasma instabilities.

In the accelerator and transport regions, the analysis in this paper assumes a long ion charge bunch (bunch length $l_{b} \gg$ bunch radius $r_{b}$ ) with directed axial kinetic energy $\left(\gamma_{b}-1\right) m_{b} c^{2}$ propagating in the $z$ direction through a perfectly conducting cylindrical pipe with constant radius $r_{w}$. The analysis is carried out in the smooth-focusing approximation where the applied transverse focusing force is modeled by $\mathbf{F}_{f o c}=-\gamma_{b} m_{b} \omega_{f}^{2} \mathbf{x}_{\perp}$. Here, $\gamma_{b}=\left(1-\beta_{b}^{2}\right)^{-1 / 2}$ is the relativistic mass factor, $V_{b}=\beta_{b} c$ is the directed axial velocity of the charged bunch, $m_{b}$ is the ion rest mass, $\omega_{f}=$ const is the single-particle oscillation frequency associated with the applied focusing force, and $\mathbf{x}_{\perp}=x \mathbf{e}_{x}+y \mathbf{e}_{y}$ is the transverse displacement of a beam particle from the cylinder axis. Denoting the characteristic number density of beam particles by $\hat{n}_{b}$ and the particle charge by $e_{b}$, it is convenient to introduce the relativistic plasma frequency $\hat{\omega}_{p b}$ defined by $\hat{\omega}_{p b}=\left(4 \pi \hat{n}_{b} e_{b}^{2} / \gamma_{b} m_{b}\right)^{1 / 2}$ and the normalized (dimensionless) beam intensity $s_{b}$ defined by $s_{b}=\hat{\omega}_{p b}^{2} / 2 \gamma_{b}^{2} \omega_{f}^{2}[8]$. Furthermore, the particle dynamics in the beam frame is assumed to be nonrelativistic.

It should be noted that one collective instability that is not summarized in the present paper is the electron-ion two-stream (electron cloud) instability, which can occur when an intense ion beam propagates through an (unwanted) partially-neutralizing component of background electrons produced (for example) when energetic beam ions strike the chamber wall or ionize background gas atoms. Advanced analytical and numerical simulation studies of this instability have previously been reported [64-70,78] and will not be repeated here, except to note that the conditions for eliminating or greatly reducing the effects of this instability have been identified, e.g., through the introduction of a very modest axial momentum spread in the beam ions.

As noted earlier, Secs. II and III provide a brief overview of the present understanding of several collective instabilities that can develop in intense ion beams and beam-plasma systems. 
While the summaries are necessarily short, the references in the bibliography for this paper provide considerable detailed information.

\section{ANISOTROPY-DRIVEN COLLECTIVE INSTABILITIES IN ONE- COMPONENT NONNEUTRAL BEAMS}

\section{A. Nonlinear Stability Theorem}

A very important consequence of the nonlinear Vlasov-Maxwell equations is the existence of a stability theorem (a sufficient conditions for stability) for a one-component charged particle beams. In particular, for a long, coasting beam in the smooth-focusing approximation, the stability theorem states that any equilibrium distribution function $f_{b}^{0}\left(H^{\prime}\right)$ that satisfies

$$
\frac{\partial}{\partial H^{\prime}} f_{b}^{0}\left(H^{\prime}\right) \leq 0
$$

is nonlinearly stable to perturbation with arbitrary polarization [8, 23, 24]. In Eq. (1), $H^{\prime}=\left(p_{r}^{\prime 2}+p_{\theta}^{\prime 2}+p_{z}^{\prime 2}\right) / 2 m_{b}+m_{b} \omega_{f}^{\prime 2} r^{\prime 2} / 2+e_{b} \phi^{0}\left(r^{\prime}\right)$ is the single-particle Hamiltonian in the beam frame (primed variables), and $\phi^{0}\left(r^{\prime}\right)$ is determined self-consistently in terms of the beam spacecharge from the Poisson's equation. It follows from Eq. (1) that any isotropic distribution that is a monotonic decreasing function of energy in the beam frame is nonlinearly stable. The validity of this theorem has been demonstrated in nonlinear $\delta f$ simulations $[67,83]$ for beam propagation over thousands of equivalent lattice periods.

Equation (1) is a sufficient condition for stability. Therefore, a necessary condition for instability is that the beam distribution function has some distinct nonthermal feature such as an inverted population in phase space [11-13], or a strongly anisotropic distribution function in the beam frame. In electrically neutral plasmas, energy anisotropies are well known to provide the free energy to drive the classical electrostatic Harris instability [32] and the electromagnetic Weibel instability [43]. The drive mechanism for instability can be either a temperature anisotropy or an anisotropy in the relative directed kinetic energy of the plasma components.

\section{B. Electrostatic Harris Instability for One-Component Beams}


In accelerators, strongly anisotropic beam distributions $T_{\| b} / T_{\perp b} \ll<1$ develop naturally during the acceleration of the charge bunch [10], and can provide the free energy to drive both the electrostatic Harris instability [32-42] and the electromagnetic Weibel instability [39, 43-48]. In this section, we summarize theoretical advances in recent analytical and numerical studies of the Harris instability in intense one-component beams [33-39, 78], assuming electrostatic perturbations $(\nabla \times \delta \mathbf{E} \cong 0$ and $\delta \mathbf{B} \cong 0)$ about an initial anisotropic thermal equilibrium distribution $\left(T_{\| b}<T_{\perp b}\right)$ described by the self-consistent Vlasov equilibrium

$$
f_{b}^{0}(r, \mathbf{p})=\frac{\hat{n}_{b}}{\left(2 \pi m_{b} T_{\perp b}\right)} \exp \left(-\frac{H_{\perp}}{T_{\perp b}}\right) \frac{1}{\left(2 \pi m_{b} T_{\| b}\right)^{1 / 2}} \exp \left(-\frac{p_{z}^{2}}{2 m_{b} T_{\| b}}\right)
$$

in the beam frame. The 'primed' notation for beam-frame variables has been dropped in Eq. (2). In Eq. (2), $H_{\perp}=p_{\perp}^{2} / 2 m_{b}+(1 / 2) m_{b} \omega_{f}^{2}\left(x^{2}+y^{2}\right)+e_{b} \phi^{0}(r)$ is the single-particle Hamiltonian for the transverse particle motion, $p_{\perp}=\left(p_{\theta}^{2}+p_{r}^{2}\right)^{1 / 2}$ is the transverse particle momentum, $r=\left(x^{2}+y^{2}\right)^{1 / 2}$ is the radial distance from the beam axis, and $\phi^{0}(r)$ is determined selfconsistently from the equilibrium Poisson equation $r^{-1}(\partial / \partial r)\left[r \partial \phi^{0} / \partial r\right]=-4 \pi e_{b} \int d^{3} p f_{b}^{0}(r, \stackrel{p}{p})$. In the remainder of Sec. II, it is convenient to introduce the effective depressed betatron frequency $\omega_{\beta \perp}$ defined by [37]

$$
\omega_{\beta \perp}^{2}=\frac{2 T_{\perp b}}{m_{b} r_{b}^{2}}=\omega_{f}^{2}-\bar{\omega}_{p b}^{2} / 2,
$$

where $T_{\perp b}$ is the transverse beam temperature, $r_{b}$ is the root-mean-square beam radius, $m_{b}$ is the mass of a beam particle, and

$$
\bar{\omega}_{p b}^{2}=\frac{4 \pi e_{b}^{2}}{m_{b} r_{b}^{2}} \int_{0}^{r_{w}} d r r n_{b}(r)
$$

is the average beam plasma frequency-squared. The normalized tune depression $\bar{v} / v_{0}$ is defined by

$$
\frac{\bar{v}}{v_{0}}=\frac{\omega_{\beta \perp}}{\omega_{f}},
$$

where $\omega_{f}=$ const is the transverse frequency associated with the applied focusing field in the smooth-focusing approximation. 
Detailed 3D numerical studies of the electrostatic Harris instability in intense onecomponent beams have been carried out using the linear eigenmode code bEASt and the nonlinear $\delta f$ simulations code BEST, and detailed results are presented in [33-39] and [78]. For

present purpose we summarize here some of the most important results.

Shown in Fig. 1 are plots of the normalized growth rate $(\operatorname{Im} \omega)_{\max } / \omega_{f}$ versus the normalized tune depression $\bar{v} / v_{0}$ obtained for $T_{\| b} / T_{\perp b}=0$ and wall radius $r_{w}=3 r_{b}$ [39]. The dashed curve in Fig. 1 has been calculated using the eigenmode code bEASt [37,39] for perturbations with azimuthal mode number $m=1$, which has the largest growth rate. The solid curve in Fig. 1 [39] is obtained from the simplified approximate dispersion relation [37]

$$
\frac{1}{1-\bar{v}^{2} / v_{0}^{2}}=\frac{1}{\left(\omega / \omega_{f}-\bar{v} / v_{0}\right)^{2}}+\frac{1}{\left(\omega / \omega_{f}+\bar{v} / v_{0}\right)^{2}}
$$

The $m=1$ dipole mode is purely growing with $\operatorname{Re} \omega=0$. Note from the dashed curve in Fig. 1 that $(\operatorname{Im} \omega)_{\max } / \omega_{f} \cong 0.34$ for $\bar{v} / v_{0} \cong 0.62$, and that the Harris instability is completely absent for $\bar{v} / v_{0}>0.82$, corresponding to sufficiently small tune shift that $(\delta \bar{v}) / v_{0}=\left(v_{0}-\bar{v}\right) / v_{0}<0.18$.

The nonlinear $\delta f$ code BEST has been used to follow the detailed nonlinear evolution and saturation of the Harris instability [33-39]. Shown in Fig. 2 are plots versus $\bar{v} / v_{0}$ of the threshold values of the anisotropy $T_{\| b} / T_{\perp b}$ required for complete stabilization of the Harris instability [39]. The dashed (solid) curves in Fig. 2 correspond to threshold values of $T_{\| b} / T_{\perp b}$ for azimuthal mode numbers $\mathrm{m}=0,1$ obtained from the linear eigenmode code bEASt. The squares in Fig. 2 correspond to the effective longitudinal beam temperature $T_{\| b} \equiv m_{b}\left\langle v_{\|}^{2}\right\rangle$ obtained in nonlinear $\delta f$ simulations after the instability saturates. As discussed in [37], the Harris instability in a one-component beam saturates nonlinearly through a combination of particle trapping and quasilinear relaxation.

Two important conclusions are evident from Figs. 1 and 2. The beam parameters in many accelerator systems satisfy $\bar{v} / v_{0}>0.82$, in which case the Harris instability is expected to be completely absent. On the other hand, for ion-beam-driven high energy density physics and heavy ion fusion applications, the beam intensity may be sufficiently high that $\bar{v} / v_{0}<0.82$. In 
this case, it is evident from Figs. 1 and 2 that the nonlinear dynamics of the Harris instability can play an important role in increasing $T_{\| b} \equiv m_{b}\left\langle v_{\|}^{2}\right\rangle$, which could have a deleterious effect on longitudinal focusing of the beam pulse if $T_{\| b}$ increases to sufficiently large values. This would of course require a sufficiently long beam transport region for the instability to grow to a substantial level.

\section{Electromagnetic Weibel Instability for One-Component Beams}

The eigenmode code bEASt and nonlinear $\delta f$ code BEST have been extended to incorporate slow-wave transverse electromagnetic perturbations (so called Darwin model), thereby allowing for the possibility of a Weibel-type instability occurring in a one-component charged particle beam [39,43-45]. Finite-geometry and self-field effects make a precise stability analysis based on the linearized Vlasov-Maxwell equations difficult analytically. However, for a anisotropic distribution of beam particles [Eq. (2)], assuming $T_{\| b} \rightarrow 0, k_{z}^{2} r_{b}^{2}>>1$, and $\bar{\omega}_{p b}^{2} r_{b}^{2} / c^{2}<<1$, a simplified analytical model gives the simple approximate estimate [39]

$$
\frac{(\operatorname{Im} \omega)_{\max }}{\omega_{f}}=\frac{1}{\sqrt{2}} \frac{\bar{\omega}_{p b}}{\omega_{f}} \frac{v_{\perp b}^{t h}}{c}
$$

for the maximum growth rate of the Weibel instability. Here, $v_{\perp b}^{t h} \equiv\left(2 T_{\perp b} / m_{b}\right)^{1 / 2}$ is the transverse thermal speed of the beam particles. Making use of Eqs. (3) and (5), it is readily shown that Eq. (7) can be expressed in the equivalent form

$$
\frac{(\operatorname{Im} \omega)_{\max }}{\omega_{f}}=\frac{\bar{v}}{v_{0}}\left(1-\frac{\bar{v}^{2}}{v_{0}^{2}}\right)^{1 / 2} \frac{\omega_{f} r_{b}}{c} .
$$

Note from Eq. (8) that $(\operatorname{Im} \omega)_{\max }$ assumes a maximum value of $(\operatorname{Im} \omega)_{\max }=0.5 \omega_{f}^{2} r_{b} / c$ for $\bar{v} / v_{0}=1 / \sqrt{2}=0.707$ (see Fig. 3).

A typical plot of the normalized maximum growth rate $(\operatorname{Im} \omega)_{\max } /\left(\omega_{f}^{2} r_{b} / c\right)$ versus normalized tune $\bar{v} / v_{0}$ obtained numerically using the linear eigenmode code bEASt is illustrated by the solid curve in Fig. 4 [39] for the choice of system parameters $T_{\| b} / T_{\perp b}=0, r_{w}=3 r_{b}$, and $\bar{\omega}_{p b} r_{b} / c<<1$. Quite remarkably, taking $r_{w}=3 r_{b}$ and comparing Figs. 3 and 4 , the value of $\bar{v} / v_{0}$ at maximum growth is in very good agreement with the theoretical estimate in Fig. 3, and the 
corresponding value of the maximum growth rate in Fig. 4 is in agreement within a factor of two. The squares shown in Fig. 4 for $0.82<\bar{v} / v_{0}<1$ are the results obtained from simulations carried out using the linearized version of the Darwin BEST code for $T_{\| b} / T_{\perp b}=10^{-4}$ and $r_{w}=3 r_{b}$ [39]. Note that the BEST results connect smoothly to the bEASt results in Fig. 4.

From Sec. II.B. and Fig. 1 it is clear that the Harris instability can have a substantial growth rate provided by $\bar{v} / v_{0}<0.82$ and $T_{\| b} / T_{\perp b}$ is smaller than the threshold value for stabilization shown in Fig. 2. On the other hand, from Figs. 3 and 4, the electromagnetic Weibel instability typically has a very small growth rate for $\bar{v} / v_{0}<0.82$, because $\omega_{f}^{2} r_{w}^{2} / c^{2}<<1$ and $\bar{\omega}_{p b}^{2} r_{b}^{2} / c^{2}<<1$ in the parameter regimes of practical interest. In addition, the threshold value of $T_{\| b} / T_{\perp b}$ for complete stabilization of the Weibel instability is extremely small, due to finite transverse geometry effects, and can be estimated to be [37,39]

$$
\frac{T_{\| b}^{\text {th }}}{T_{\perp b}} \cong 2 \times 10^{-0.7} \frac{\bar{\omega}_{p b}^{2} r_{b}^{2}}{c^{2}}<<1 .
$$

As a consequence, the Weibel instability is much less dangerous for intense beams with normalized tune $\bar{v} / v_{0}<0.82$. This is because such intense beams are strongly unstable to the electrostatic Harris instability, which saturates at a much larger longitudinal temperature, $\left(T_{\| b}^{\text {th }} / T_{\perp b}\right)^{\text {Weibel }}<<\left(T_{\| b}^{\text {th }} / T_{\perp b}\right)^{\text {Harris }} \approx 0.11$, and has a much larger growth rate,

$\gamma_{\text {Weibel }} / \gamma_{\text {Harris }} \sim v_{\perp b}^{\text {th }} / c \ll 1$, where $\gamma_{\text {Weibel }}$ denotes $(\operatorname{Im} \omega)_{\max }$ for the Weibel instability, and $\gamma_{\text {Harris }}$ denotes $(\operatorname{Im} \omega)_{\max }$ for the Harris instability.

In summary, the electromagnetic Weibel instability is likely to be an important instability mechanism in one-component charged particle beams with $\bar{v} / v_{0}>0.82$, but not in intense beams with $\bar{v} / v_{0}<0.82$.

\section{COLLECTIVE INTERACTION PROCESSES FOR INTENSE BEAM PROPAGATION THROUGH BACKGROUND PLASMA}

The topics covered in Sec. II included a discussion of the sufficient condition for quiescent (stable) beam propagation over long distances (Sec. II. A); and the electrostatic Harristype instability (Sec. II. B) and the electromagnetic Weibel-type instability (Sec. II.C) in strongly anisotropic, one component nonneutral beams. In Sec. III, the collective processes associated 
with the interaction of an intense ion beam pulse with a large-volume, charge-neutralizing background plasma are described. To achieve the high focal spot intensities necessary for high energy density physics and heavy ion fusion applications, compression of the beam longitudinally and transversely in the presence of a dense charge-neutralizing background plasma has many attractive features [1-7], particularly because the plasma electrons eliminate (or significantly reduce) the large, defocusing space-charge force of the ion beam pulse. The collective beam-plasma interaction processes summarized in this section include: the multispecies electromagnetic Weibel instability (Sec. III.A); the electrostatic electron-ion twostream instability (Sec. III.B); the dynamic stabilization of the two-stream instability during longitudinal drift compression (Sec. III.C); and the effects of solenoidal magnetic field on collective beam-plasma instabilities (Sec. III.D).

\section{A. Multispecies Weibel Instability}

The electromagnetic Weibel instability [39, 43-48] was shown in Sec. II.C to be relatively ineffective in one-component charged particle beams. The situation can be quite different, however, when an intense beam propagates through background plasma [78-84]. In this case, the large energy anisotropy associated with the directed kinetic energy of the beam particles relative to the background plasma can provide significant free energy to drive the transverse electromagnetic Weibel instability, and cause filamentation in the plane perpendicular to beam propagation. In this section, we summarize the results of a macroscopic cold-fluid model in which an intense ion beam $(j=b)$ propagates through a background plasma $(j=e, i)$. The background plasma is assumed to provide complete charge and current neutralization with [84]

$$
\sum_{j=b, e, i} n_{j}^{0}(r) e_{j}=0, \text { and } \sum_{j=b, e, i} n_{j}^{0}(r) e_{j} \beta_{j} c=0 .
$$

In Eq. (10), $V_{z j}=\beta_{j} c$ is the average axial velocity of species $j(j=b, e, i)$, and $\gamma_{j}=\left(1-\beta_{j}^{2}\right)^{-1 / 2}$ is the relativistic mass factor. Moreover, current neutralization has been assumed since this case gives the largest growth rate for the multispecies Weibel instability. That is, a finite azimuthal self-magnetic field $B_{\theta}^{0}(r) \neq 0$ tends to reduce the growth rate of the Weibel instability [9, 47]. Furthermore, the present analysis assumes axisymmetric flute perturbations with $\partial / \partial \theta=0$ and $\partial / \partial z=0$, and electromagnetic field perturbations with components $\delta \mathbf{E}=\delta E_{r} \mathbf{e}_{r}+\delta E_{z} \mathbf{e}_{z}$ and $\delta \mathbf{B}=\delta B_{\theta} \mathbf{e}_{\theta}$. Note that the field perturbations have mixed polarization 
with both a longitudinal component $\left(\delta E_{r} \neq 0\right)$ and the transverse electromagnetic components $\left(\delta B_{\theta} \neq 0\right.$ and $\delta E_{z} \neq 0$ ). Finally, it is assumed that the beam-plasma interactions take place in a region where there is no applied focusing field $\left(\omega_{f}=0\right)$, and a perfectly conducting cylindrical wall is located at radius $r=r_{w}$. We express $\delta E_{z}(r, t)=\delta \hat{E}_{z} \exp (-i \omega t)$, where $\operatorname{Im} \omega>0$ corresponds to instability. Making use of a cold-fluid model that neglects pressure perturbations, this leads to the eigenvalue equation [79]

$$
\frac{1}{r} \frac{\partial}{\partial r}\left[r\left[1+\sum_{j=b, e, i} \frac{\beta_{j}^{2} \omega_{p j}^{2}(r)}{\omega^{2}}+\sum_{j=b, e, i} \frac{\left[\beta_{j} \omega_{p j}^{2}(r)\right]^{2}\left[\omega^{2}-\sum_{j=b, e, i} \omega_{p j}^{2}(r)\right]}{\partial r} \delta E_{z}\right]+\left(\frac{\omega^{2}}{c^{2}}-\sum_{j=b, e, i} \frac{\omega_{p j}^{2}(r)}{\gamma_{j}^{2} c^{2}}\right) \delta E_{z}=0,\right.
$$

where $\omega_{p j}(r)=\left[4 \pi n_{j}^{0}(r) e_{j}^{2} / \gamma_{j} m_{j}\right]^{1 / 2}$ and $\gamma_{j}=\left(1-\beta_{j}^{2}\right)^{-1 / 2}$.

Equation (11) is the desired eigenvalue equation, with the terms proportional to $\sum_{j=b, e, i} \beta_{j}^{2} \omega_{p j}^{2}(r)$ and $\sum_{j=b, e, i} \beta_{j} \omega_{p j}^{2}(r) \neq 0$ providing the free energy to drive the multispecies Weibel instability. Equation (11) can be integrated numerically to determine the eigenvalue $\omega^{2}$ and eigenfunction $\delta \hat{E}_{z}(r)$ for a wide range of beam-plasma density profiles $n_{j}^{0}(r)$. Analytical solutions are also tractable for the case of flat-top (step-function) density profiles [79]. As a general remark, when $\sum_{j=b, e, i} \beta_{j}^{2} \omega_{p j}^{2}(r) \neq 0$ and $\sum_{j=b, e, i} \beta_{j} \omega_{p j}^{2}(r) \neq 0$, Eq. (11) supports both stable fast-wave solutions with $\operatorname{Im} \omega=0,\left|\omega / c k_{\perp}\right|>1$, and unstable slow-wave solutions with $\operatorname{Im} \omega>0,\left|\omega / c k_{\perp}\right|<1$ [79]. Here, $\left|k_{\perp} \sim \partial / \partial r\right|$ is the characteristic radial wave number of the perturbation. Equation (11) also supports plasma oscillation solutions associated with the factor proportional to $\left[\omega^{2}-\sum_{j=b, e, i} \omega_{p j}^{2}(r)\right]^{-1}$.

As an example that is analytically tractable, we consider the case where the density profiles are uniform both inside and outside the beam (Fig. 5) with $n_{j}^{0}(r)$ specified by

$$
n_{j}^{0}(r)=\hat{n}_{j}^{i}=\text { const }, \quad j=b, e, i
$$

for $0 \leq r<r_{b}$, and

$$
n_{j}^{0}(r)=\hat{n}_{j}^{o}=\text { const }, \quad j=e, i
$$


for $r_{b}<r \leq r_{w}$. The transcendental dispersion relation derived from Eq. (11) for step-function density profiles has been solved numerically $[79,84]$ for the complex oscillation frequency $\omega$ for a wide range of system parameters corresponding to (a) plasma-filled waveguide $\left(r_{b}=r_{w}\right)$; (b) plasma outside the beam-plasma channel $\left(\hat{n}_{j}^{o} \neq 0, j=e, i\right.$, and $\left.r_{b}<r_{w}\right)$; and (c) no plasma outside the beam-plasma channel $\left(\hat{n}_{j}^{o}=0, j=e, i\right.$, and $\left.r_{b}<r_{w}\right)$. Assuming a positively charged ion beam $(j=b)$ propagating through background plasma electrons and ions $(j=e, i)$, the charge states are denoted by $e_{b}=+Z_{b} e, e_{e}=-e$, and $e_{i}=+Z_{i} e$, and the plasma electrons are assumed to carry the neutralizing current $\left(\beta_{e} \neq 0\right)$, whereas the plasma ions are taken to be stationary $\left(\beta_{i}=0\right)$. The conditions for charge neutralization and current neutralization, in Eq. (10) then give

$$
\begin{aligned}
& \hat{n}_{e}^{i}=Z_{b} \hat{n}_{b}^{i}+Z_{i} \hat{n}_{i}^{i}, \\
& \beta_{e}=\frac{\beta_{b} Z_{b} \hat{n}_{b}^{i}}{Z_{b} \hat{n}_{b}^{i}+Z_{i} \hat{n}_{i}^{i}} .
\end{aligned}
$$

In the analysis of the dispersion relation [79], it is useful to define

$$
\begin{array}{r}
\Omega_{p}^{i 2} \equiv \sum_{j=b, e, i} \hat{\omega}_{p j}^{i 2}, \quad \Omega_{p}^{o 2} \equiv \sum_{j=e, i} \hat{\omega}_{p j}^{o 2}, \\
\left\langle\beta^{2}\right\rangle \equiv \frac{\sum_{j=b, e, i} \beta_{j}^{2} \hat{\omega}_{p j}^{i 2}}{\sum_{j=b, e, i} \hat{\omega}_{p j}^{i 2}},\langle\beta\rangle \equiv \frac{\sum_{j=b, e, i} \beta_{j} \hat{\omega}_{p j}^{i 2}}{\sum_{j=b, e, i} \hat{\omega}_{p j}^{i 2}},
\end{array}
$$

where $\hat{\omega}_{p j}^{i 2}=4 \pi \hat{n}_{j}^{i} e_{j}^{2} / \gamma_{j} m_{j}, \gamma_{j}=\left(1-\beta_{j}^{2}\right)^{-1 / 2}$, and $\hat{\omega}_{p j}^{o 2}=4 \pi \hat{n}_{j}^{i} e_{j}^{2} / \gamma_{j} m_{j}$. Note from Eq. (15) that $\sum_{j=b, e, i} \hat{\omega}_{p j}^{i 2} / \gamma_{j}^{2} \equiv \Omega_{p}^{i 2}-\left\langle\beta^{2}\right\rangle \Omega_{p}^{i 2}$. Careful examination of the dispersion relation [79] for shortwavelength radial perturbations shows that the growth rate $\operatorname{Im} \omega$ of the unstable Weibel solution scales like $\Gamma_{W}$, where

$$
\Gamma_{W}^{2} \equiv\left[\left\langle\beta^{2}\right\rangle-\langle\beta\rangle^{2}\right] \Omega_{p}^{i 2}=\frac{\left(\beta_{e}^{2} \hat{\omega}_{p e}^{i 2}+\beta_{b}^{2} \hat{\omega}_{p b}^{i 2}\right) \hat{\omega}_{p i}^{i 2}+\left(\beta_{b}-\beta_{e}\right)^{2} \hat{\omega}_{p e}^{i 2} \hat{\omega}_{p b}^{i 2}}{\sum_{j=b, e, i} \hat{\omega}_{p j}^{i 2}}
$$

for $\beta_{i}=0$. For $\hat{\omega}_{p b}^{i 2}, \hat{\omega}_{p i}^{i 2} \ll<\hat{\omega}_{p e}^{i 2}$, it follows that Eq. (16) is given to good approximation by

$$
\Gamma_{W}^{2}=\beta_{e}^{2} \hat{\omega}_{p i}^{i 2}+\left(\beta_{b}-\beta_{e}\right)^{2} \omega_{p b}^{i 2} .
$$


Note from Eq. (17) that $\Gamma_{W}$ involves the plasma frequencies of both the beam ions and the plasma ions.

The transcendental dispersion relation obtained from Eq. (11) has been solved numerically for the complex eigenfrequency $\omega$ and eigenfunction $\delta \hat{E}_{z}(r)$ for a wide range of system parameters [79, 84]. Typical numerical results for the unstable slow-wave (Weibel) branch are illustrated in Figs. 6 and 7 for the choice of system parameters $r_{w}=3 r_{b}, \beta_{b}=0.2$, $\beta_{e}=0.1, \hat{n}_{i}^{i}=\hat{n}_{e}^{i} / 2=\hat{n}_{b}^{i}=\hat{n}_{e}^{o}=\hat{n}_{i}^{o}$, and $\Omega_{p}^{i} r_{b} / c=1 / 3$ (Fig. 6) and $\Omega_{p}^{i} r_{b} / c=3$ (Fig. 7). Here, it is also assumed that $\beta_{e}=0=\beta_{i}$ in the region outside the beam-plasma channel, and that the plasma ions are stationary $\left(\beta_{i}=0\right)$ inside the channel. Shown in Figs 6 and 7 are plots of the normalized growth rate $\operatorname{Im} \omega / \Gamma_{W}$ versus radial mode number $n$, and plots of the eigenfunction $\delta \hat{E}_{z}(r)$ versus $r / r_{w}$ for mode number $n=5$. It is evident from Figs. 6 and 7 that $\Gamma_{W}$ [Eq. (17)] gives a very good estimate of the maximum growth rate of the multispecies Weibel instability.

To summarize, the multispecies Weibel instability with characteristic growth rate $\Gamma_{W}$ can be particularly virulent for a sufficiently intense (high density) ion charge bunch propagating through background plasma that provides complete charge and current neutralization. On the other hand, the multispecies Weibel instability is not expected to have a deleterious effect on the beam quality provided

$$
\Gamma_{W} \tau_{p}<1,
$$

where $\tau_{p}=L_{p} / V_{b}$ is the interaction time of the beam ions with background plasma, and $L_{p}$ is the length of the plasma column. Equivalently, $\Gamma_{W} \tau_{p}<1$ gives

$$
L_{p}<\alpha \frac{c}{\hat{\omega}_{p b}^{i}}=2.3 \times 10^{7} \alpha \frac{A_{b}^{1 / 2}}{\left[\hat{n}_{b}^{i}\left(\mathrm{~cm}^{-3}\right)\right]^{1 / 2}} \mathrm{~cm},
$$

where use is made of Eq. (17), and the constant $\alpha$ is defined in the nonrelativistic case by

$$
\alpha=\left[\left(1-\frac{Z_{b} \hat{n}_{b}^{i}}{\hat{n}_{e}^{i}}\right)^{2}+\frac{Z_{i}}{Z_{b}} \frac{m_{b}}{m_{i}} \frac{Z_{b} \hat{n}_{b}^{i}}{\hat{n}_{e}^{i}}\left(1-\frac{Z_{b} \hat{n}_{b}^{i}}{\hat{n}_{e}^{i}}\right)\right]^{-1 / 2} .
$$

For singly ionized Aluminum beam ions $\left(Z_{b}=1\right.$ and $\left.A_{b}=13\right)$ in background Argon plasma $\left(A_{i}=18\right.$ ) and $\hat{n}_{b}^{i} / \hat{n}_{e}^{i}=1 / 2$, we obtain from Eqs. (19) and (20) that $L_{p}<1.27 \mathrm{~m}, 12.7 \mathrm{~m}$, for 
$\hat{n}_{b}^{i}=10^{12} \mathrm{~cm}^{-3}, 10^{10} \mathrm{~cm}^{-3}$. Therefore the exponential length for the multispecies Weibel instability is moderately long, even for beam densities in the range $10^{10} \mathrm{~cm}^{-3}-10^{12} \mathrm{~cm}^{-3}$.

\section{B. Electrostatic Two-Stream Instability}

The relative streaming of the beam ions through the background plasma components can also provide the free energy to drive the electrostatic two-stream instability with characteristic polarization $\nabla \times \delta \mathbf{E} \cong 0$ and $\delta \mathbf{B} \cong 0$. In this section, we make similar assumptions to those made at the beginning of Sec. III.A, including equilibrium charge and current neutralization [Eq. (10)], absence of an applied focusing field $\left(\omega_{f}=0\right)$, and a perfectly conducting cylindrical wall located at radius $r=r_{w}$. Expressing the longitudinal electric field perturbations as $\delta \mathbf{E}=-\nabla \delta \phi$, we assume axisymmetric perturbations with $\partial / \partial \theta=0$. Perturbed quantities are expressed as $\delta \phi(r, z, t)=\delta \hat{\phi}(r) \exp \left[i\left(k_{z} z-\omega t\right)\right]$, where $k_{z}$ is the axial wave number, and $\operatorname{Im} \omega>0$ corresponds to instability (temporal growth). Without presenting algebraic details [84, 85], the linearized cold-fluid equations lead to the electrostatic eigenvalue equation

$$
\frac{1}{r} \frac{\partial}{\partial r}\left[r\left(1-\sum_{j=b, e, i} \frac{\omega_{p j}^{2}(r) / \gamma_{j}^{2}}{\left(\omega-k_{z} V_{z j}\right)^{2}}\right) \frac{\partial}{\partial r} \delta \hat{\phi}\right]-k_{z}^{2}\left(1-\sum_{j=b, e, i} \frac{\omega_{p j}^{2}(r) / \gamma_{j}^{2}}{\left(\omega-k_{z} V_{z j}\right)^{2}}\right) \delta \hat{\phi}=0 .
$$

Here $\omega_{p j}(r)=\left[4 \pi n_{j}^{0}(r) e_{j}^{2} / \gamma_{j} m_{j}\right]^{1 / 2}$ is the relativistic plasma frequency, $V_{z j}=\beta_{j} c=$ const is the average axial velocity of component $j(j=b, e, i)$, and $\gamma_{j}=\left(1-\beta_{j}^{2}\right)^{-1 / 2}$ is the relativistic mass factor.

The electrostatic eigenvalue equation (22) can be solved numerically for the eigenfunction $\delta \hat{\phi}(r)$ and the complex eigenfrequency $\omega$ for a wide range of density profiles $n_{j}^{0}(r)(j=b, e, i)$. For present purposes, we specialize again to the choice of flat-top density profiles in Eqs. (12) and (13). In this case, the eigenfunction $\delta \hat{\phi}(r)$ can be determined analytically in the beam-plasma channel $\left(0 \leq r<r_{b}\right)$, and in the region outside the beam $\left(r_{b}<r \leq r_{w}\right)$. Employing the appropriate boundary conditions at $r=r_{b}$, and enforcing $\delta \hat{\phi}\left(r=r_{w}\right)=0$, some straightforward algebra leads to the electrostatic dispersion relation $[78,84]$

$$
D\left(k_{z}, \omega\right)=1-g_{0} \sum_{j=b, e, i} \frac{\hat{\omega}_{p j}^{i 2} / \gamma_{j}^{2}}{\left(\omega-k_{z} V_{z j}\right)^{2}}-\left(1-g_{0}\right) \sum_{j=e, i} \frac{\hat{\omega}_{p j}^{o 2} / \gamma_{j}^{2}}{\omega^{2}}=0 .
$$


Here, the geometric factor $g_{0}$ is defined by

$$
g_{0}=k_{z} r_{b} I_{0}^{\prime}\left(k_{z} r_{b}\right) I_{0}\left(k_{z} r_{b}\right)\left[\frac{K_{0}\left(k_{z} r_{b}\right)}{I_{0}\left(k_{z} r_{b}\right)}-\frac{K_{0}\left(k_{z} r_{w}\right)}{I_{0}\left(k_{z} r_{w}\right)}\right]
$$

for $r_{b} \neq r_{w}$, where $I_{0}(x)$ and $K_{0}(x)$ are the modified Bessel functions of the first and second kinds, respectively, of order zero, and $I_{0}^{\prime}(x)$ denotes $(d / d x) I_{0}(x)$. In addition, $\hat{\omega}_{p j}^{i}=\left(4 \pi \hat{n}_{j}^{i} e_{j}^{2} / \gamma_{j} m_{j}\right)^{1 / 2}(j=b, e, i)$ is the $j^{\text {th }}$ component plasma frequency inside the beam-plasma channel $\left(0 \leq r<r_{b}\right)$, and $\hat{\omega}_{p j}^{o}=\left(4 \pi \hat{n}_{j}^{o} e_{j}^{2} / \gamma_{j} m_{j}\right)^{1 / 2}$ is the $j^{\text {th }}$ component plasma frequency outside the beam-plasma channel $\left(r_{b}<r \leq r_{w}\right)$. It is also assumed that the plasma ions are stationary $\left(\beta_{i}=0\right)$ inside the channel. The conditions for charge neutralization and current neutralization in the beam-plasma channel then reduce to Eq. (14). Finally, note from Eq. (23) that the geometric factor $g_{0}$ exhibits a strong dependence on axial wave number $k_{z}$, with

$$
\begin{gathered}
g_{0} \cong \frac{1}{2} k_{z}^{2} r_{b}^{2} \ln \left(\frac{r_{w}}{r_{b}}\right), \text { for } k_{z}^{2} r_{w}^{2}<<1, \\
g_{0} \cong \frac{1}{2}, \text { for } k_{z}^{2} r_{w}^{2}>>1 .
\end{gathered}
$$

Because of the geometric factors $g_{0}$ and 1- $g_{0}$, the detailed properties of the two-stream instability calculated from Eq. (22) can differ substantially from the infinite beam-plasma results. However, several interesting features are evident. First, in the absence of plasma outside the beamplasma channel $\left(\hat{\omega}_{p j}^{o 2}=0\right)$, the channel electrons undergo unstable two-stream interactions with both the beam ions and the channel plasma ions. Second, when there is plasma outside the beamplasma channel $\left(\hat{\omega}_{p j}^{o 2} \neq 0\right.$ ), the channel electrons can also undergo a strong unstable two-stream interaction with the plasma electrons outside the channel. Illustrative solutions to the dispersion relation (22) are presented in [78, 84]. For present purposes, we consider the case when there is no plasma outside the beam-plasma channel, i.e., $\hat{n}_{e}^{o}=0=\hat{n}_{i}^{o}$ for $r_{b}<r \leq r_{w}$, and assume a cesium ion beam with $\beta_{b}=0.2$ and $Z_{b}=1$ propagating through background argon plasma with $Z_{i}=1$ and $\beta_{i}=0$. The current neutralization condition in Eq. (14) then gives $\beta_{e}=0.1$. The dispersion relation (22) has two unstable branches corresponding to the interaction of the plasma electrons with the beam ions, and the interaction of the plasma electrons with the plasma ions. The unstable branch illustrated in Fig. 8 corresponds to the interaction of the plasma electrons with 
the plasma ions. Figure 8 shows plots of the normalized growth $(\operatorname{Im} \omega) / \hat{\omega}_{p e}^{i}$ and real oscillations frequency $(\operatorname{Re} \omega) / \hat{\omega}_{p e}^{i}$ versus $k_{z} r_{b}$ for the case corresponding to $\hat{\omega}_{p e}^{i} r_{b} / c=3$ and $r_{b} / r_{w}=1 / 3$. Note that the two-stream growth rate is strongly peaked as a function of $k_{z} r_{b}$. For the choice of system parameters in Fig. 8, the value of $k_{z}=k_{z m}$ at maximum growth rate satisfies $k_{z m}^{2} r_{b}^{2}>>1$. In this case, $g_{0}\left(k_{z m}\right) \cong 1 / 2$ in Eq. (22), and the maximum growth rate $(\operatorname{Im} \omega)_{\max }$ and value of $k_{z m}$ in Fig. 8 are given to excellent approximation by the analytical estimates.

$$
\begin{gathered}
(\operatorname{Im} \omega)_{\max } \cong\left(\frac{3}{8}\right)^{1 / 2}\left(\frac{\hat{\omega}_{p i}^{i 2}}{2 \hat{\omega}_{p e}^{i 2}}\right)^{1 / 3} \hat{\omega}_{p e}^{i}, \\
\left|k_{z m}\right| r_{b} \cong \frac{1}{(2)^{1 / 2}} \frac{\hat{\omega}_{p e}^{i} r_{b}}{c} \frac{1}{\left|\beta_{i}-\beta_{e}\right|},
\end{gathered}
$$

where $\beta_{i}=0$ is assumed. Equation (25) corresponds to the unstable plasma electron - plasma ion two-stream solution to Eq. (22). For the unstable plasma electron - beam ion solution, the estimates are similar to those in Eq. (25) with $\hat{\omega}_{p i}^{i}$ replaced by $\hat{\omega}_{p b}^{i}$, and $\beta_{i}-\beta_{e}$ replaces by $\beta_{b}-\beta_{e}$

To summarize, for a cold ion beam propagating through a cold background plasma, the electrostatic two-stream instability can be an important collective interaction mechanism. Since the phase velocities of the most unstable modes are close to the beam velocity $\beta_{b} c$ and the plasma ion velocity $\beta_{i} c$, modest axial velocity spreads in the beam ions and plasma ions can lead to a growth rate reduction. It is also expected that somewhat rounded density profiles, rather than flat-top profiles, or a radial shear in the axial velocity profile, would result in lower instability growth rates. An important nonlinear consequence of the two-stream instability is the rapid nonlinear heating of the plasma electrons on a time scale of a few times $(\operatorname{Im} \omega)_{\max }^{-1}$. The time scale $(\operatorname{Im} \omega)_{\max }^{-1}$ can be relatively fast for the electrostatic two-stream instability. The condition for negligible two-stream interaction over a length $L_{p}$ of the plasma column can be expressed as $(\operatorname{Im} \omega)_{\max } L_{p} / V_{b}<1$, or equivalently,

$$
L_{p}<\alpha_{e s}\left(\frac{8}{3}\right)^{1 / 2} \frac{c}{\hat{\omega}_{p e}^{i}} \beta_{b},
$$


where

$$
\alpha_{e s}=\left[\left(1-\frac{Z_{b} \hat{n}_{b}^{i}}{\hat{n}_{e}^{i}}\right) \frac{Z_{i} m_{e}}{2 m_{i}}\right]^{-1 / 3}
$$

for the electron-ion two-stream growth rate estimate in Eq. (25). Here, use has been made of Eq. (14) to eliminate $\hat{n}_{i}^{i} / \hat{n}_{e}^{i}$ in favor of $\hat{n}_{b}^{i} / \hat{n}_{e}^{i}$. As a numerical example, we consider singly-ionized Aluminun beam ions $\left(Z_{b}=1\right.$ and $\left.A_{b}=13\right)$ with $\beta_{b}=0.1$ propagating through background Argon plasma $\left(A_{i}=18\right)$ with $\hat{n}_{b}^{i} / \hat{n}_{e}^{i}=1 / 2$, we obtain from Eqs. (26) and (27) that $L_{p}<31 \mathrm{~cm}$, $3.1 \mathrm{~cm}$, for $\hat{n}_{b}^{i}=10^{10} \mathrm{~cm}^{-3}, 10^{12} \mathrm{~cm}^{-3}$, which correspond to relatively short distances.

\section{Dynamic Stabilization of Two-Stream Instability During Longitudinal Beam}

\section{Compression}

Detailed properties of the electrostatic two-stream instability can change substantially during longitudinal compression of the beam pulse from those reported in Sec. III. B. In a recent calculation [81], the electrostatic two-stream instability for a cold, longitudinally-compressing intense ion beam propagating through a dense background plasma has been investigated both analytically and numerically using a simple one-dimensional model in which transverse spatial variations are neglected. The linear development of the instability and its saturation are examined from the point of view of wave dynamics, where the plasma waves are represented as quasiparticles characterized by their position $z(t)$, wavenumber $k(t)$, and energy (or frequency) $\omega(t)$. It is found that the longitudinal beam compression strongly modifies the space-time development of the instability. In particular, the dynamic compression of the beam pulse leads to a significant reduction in the growth rate of the two-stream instability compared to the case without an initial velocity tilt [81].

To briefly describe the theoretical model [81], a semi-infinite ion beam with (see Fig. 9) sharp leading edge enters the region containing background plasma at time $t=0$ and $z=0$ with velocity $V_{b}^{0}$ and density $n_{b}^{0}$. The beam is uniformly compressing in the longitudinal direction as it propagates inside the plasma and reaches the maximum compression at time $t=T_{f}$ at the point $z=Z_{f}=T_{f} V_{b}^{0}$ away from the beam entry point at $z=0$ into the chamber. The unperturbed beam propagation is illustrated in Fig. 9, where the beam phase space is plotted at different times during the compression. The transition from the solid to dashed lines in Fig. 9 identifies the end 
of the real beam pulse with finite initial length $L_{b}^{0}$. The longitudinal "velocity tilt" $\Delta V_{b}^{0} / V_{b}^{0}$, is related to the compression distance $Z_{f}$ and the initial beam pulse length $L_{b}^{0}$ by

$$
\Delta V_{b}^{0} / V_{b}^{0}=L_{b}^{0} / Z_{f}
$$

It is also assumed that the ion beam propagation in the background plasma is both charge neutralized and current neutralized, where the quasi-neutrality conditions are given by [81]

$$
\begin{gathered}
n_{e}=Z_{b} n_{b}+\hat{n}_{i}, \\
n_{e} V_{e}=Z_{b} V_{b} n_{b} .
\end{gathered}
$$

In Eq. (29) $n_{j}$ and $V_{j}$ denote the dynamically changing unperturbed density and flow velocity of the beam ions $(j=b)$ and background plasma electrons $(j=e)$, and $\hat{n}_{i}=$ const (independent of $z$ and $t$ ) is the uniform density of the background plasma ions (assumed singly ionized and immobile). In Eq. (29), $Z_{b}$ is the charge state of the beam ions.

The analysis in [81] makes use of an elegant quasi-particle formalism, and assumes two small parameters

$$
\varepsilon \equiv 1 /\left(\omega_{p e} T_{f}\right)<<1 \text { and } \delta \equiv Z_{b} n_{b}^{0} / \hat{n}_{i}<<1
$$

It is found that the two-stream instability between the beam and plasma electrons develops and saturates everywhere in the background plasma region except close to the compression point $z=Z_{f}$ during the time interval when $1-t / T_{f} \sim 1>Z_{b} n_{b}^{0} / \hat{n}_{i}$. It is convenient to introduce the gain function $G(z, t)$ defined by

$$
G(z, t)=\int_{z / V_{b}}^{t} \operatorname{Im} \omega(z, \bar{t}) d \bar{t} .
$$

Typical numerical results obtained from the linear dispersion relation and the quasi-particle dynamical equations are illustrated in Figs. 10 and 11 [81]. Figure 10 shows the normalized instability gain function $G(z, t) / \alpha$ plotted as a function of distance $z / Z_{f}$ at different times $t / T_{f}=0.15(1), 0.25(2), 0.35$ (3), $0.45(4), 0.55(5), 0.65$ (6), and 0.75 (7) obtained numerically using the quasi-particle formalism, and compared with the analytical estimate (dashed curve) [81]. Figure 11 shows a comparison of the gain function in Eq. (31) with the gain function for a beam with zero tilt velocity, i.e., with the expression 


$$
G_{\text {notilt }}\left(\frac{z}{Z_{f}}, t=T_{f}\right)=\alpha \frac{3 \sqrt{3}}{4} \frac{\left(z / Z_{f}\right)^{2 / 3}\left(1-z / Z_{f}\right)^{1 / 3}}{\delta^{1 / 6}}
$$

In Fig. 11, we have chosen system parameters to be $\delta \equiv Z_{b} n_{b}^{0} / \hat{n}_{i}=10^{-3}$ and $\alpha^{2}=\left(\omega_{p b} T_{f}\right)^{2}=1000$. For $\delta^{1 / 6} \ll 1$, it is evident from Fig. 11 that the velocity tilt significantly reduces the growth rate compared to the case of a beam with zero velocity tilt [81].

\section{Effects of Solenoidal Magnetic Field}

As noted earlier, to achieve maximum compression, the space charge of the ion beam is neutralized by the propagation of the ion beam pulse through a dense background plasma [1-7, 72-77]. In one approach, transverse compression is facilitated by using solenoidal focusing magnets. Recent studies of the beam's charge and current neutralization in plasma with solenoidal magnetic field have shown that when the magnetic field is strong enough that $\omega_{c e} \sim \beta_{b} \omega_{p e}$, the electron dynamics becomes significantly affected by the magnetic field. Specifically, if the condition $\omega_{c e} \sim \beta_{b} \omega_{p e}$ is satisfied, the magnetic field causes the plasma electrons to start rotating about the solenoid axis as they flow into the ion beam pulse to neutralize its charge and current [3]. Moreover, if $\omega_{c e}>\beta_{b} \omega_{p e}$, low frequency helicon waves propagating nearly perpendicular to the beam propagation direction can now be resonantly excited by the beam [3], drastically changing the way current is being neutralized by the background plasma. Coupling to the helicon waves also modifies the electromagnetic Weibel instability discussed in Sec. III. A in the absence of applied focusing field. In a recent calculation [80], we have studied the low-frequency electromagnetic and electrostatic streaming instabilities of an intense ion beam propagating through background plasma along a solenoidal magnetic field. Because of the large ion mass, instabilities involving the ion cyclotron motion are very slow. Therefore, in the present analysis the effect of the solenoidal magnetic field on the beam ions and plasma ions is neglected, but its effect on the plasma electrons is included in the analysis.

For present purposes, we treat the beam-plasma medium as infinite in spatial extent. The externally-applied magnetic field $B_{0} \mathbf{e}_{z}$ is directed along the $z$-direction, and the wavenumber $\mathbf{k}=k_{\perp} \mathbf{e}_{x}+k_{z} \mathbf{e}_{z}$ of the field perturbation is taken to be in the $(x, z)$ plane. Similar to previous 
sections, it is assumed that the background plasma electrons provide full charge and current neutralization, which requires the density of electrons to be $\hat{n}_{e}=Z_{i} \hat{n}_{i}+Z_{b} \hat{n}_{b}$, and the electron drift velocity to be $V_{e}=Z_{b} V_{b} \hat{n}_{b} / \hat{n}_{e}$, where $\hat{n}_{j}$ and $\hat{Z}_{j}$ are the number density and charge state of the background plasma ions $(j=i)$ and the beam ions $(j=b)$. For simplicity, the analysis [80] is carried out in a reference frame moving axially with the electrons. In this frame $\bar{V}_{b}=V_{b}-V_{e}$, $\bar{V}_{e}=0$ and $\bar{V}_{i}=-V_{e}$. Neglecting, the cyclotron motion of the beam ions and plasma ions, the full cold-plasma dispersion relations for an ion beam propagating with velocity $V_{b}$ along the magnetic field $\dot{B}_{0}$ is derived and analyzed in detail in [80].

For present purposes, we consider the case of nearly transverse propagation and lowfrequency perturbations satisfying

$$
\cos ^{2} \theta=k_{z}^{2} / k^{2}<<1,|\omega|<<\omega_{p e}, \omega_{c e},
$$

where $k^{2}=k_{\perp}^{2}+k_{z}^{2}, \quad \omega_{p e}=\left(4 \pi \hat{n}_{e} e^{2} / m_{e}\right)^{2}$, and $\omega_{c e}=e B_{0} / m_{e} c$. In addition, we consider perturbations with sufficiently short wavelength that $c^{2} k^{2} / \omega_{p e}^{2}>>1$. Making use of these approximations, the full dispersion relation derived in [80] can be approximated by

$$
\begin{aligned}
& 1+\frac{\omega_{p e}^{2}}{\omega_{c e}^{2}}\left[1+\frac{\bar{\beta}_{i}^{2} \omega_{p i}^{2}}{\left(\omega-k_{z} \bar{V}_{i}\right)^{2}}+\frac{\bar{\beta}_{b}^{2} \omega_{p b}^{2}}{\left(\omega-k_{z} \bar{V}_{b}\right)^{2}}\right]-\frac{\omega_{p e}^{2} \cos ^{2} \theta}{\omega^{2}} \\
& -\frac{\omega_{p b}^{2}}{\left(\omega-k_{z} \bar{V}_{b}\right)^{2}}-\frac{\omega_{p i}^{2}}{\left(\omega-k_{z} \bar{V}_{i}\right)^{2}}=\frac{\omega_{p i}^{2} \omega_{p b}^{2}\left(\bar{\beta}_{b}-\bar{\beta}_{i}\right)^{2}}{\left(\omega-k_{z} \bar{V}_{b}\right)^{2}\left(\omega-k_{z} \bar{V}_{i}\right)^{2}},
\end{aligned}
$$

where $\bar{\beta}_{j}=\bar{V}_{j} / c \quad(j=i, b)$. The terms proportional to $\bar{\beta}_{j}^{2}$ in Eq. (34) describe transverse electromagnetic contributions that drive the multispecies Weibel instability, whereas the remaining terms in Eq. (34) represent electrostatic two-stream contributions.

It is evident that even the simplified dispersion relation in Eq. (34) has a rich physics content that depends sensitively on the dimensionless parameter $\beta_{b}^{2} \omega_{p e}^{2} / \omega_{c e}^{2}$. For example, for $k_{z}=0$, in the limit of a weak solenoidal magnetic field with $\beta_{b}^{2} \omega_{p e}^{2} / \omega_{c e}^{2} \gg>1$, it follows that Eq. (34) can be approximated by

$$
1=-\frac{1}{\omega^{2}}\left(\bar{\beta}_{i}^{2} \omega_{p i}^{2}+\bar{\beta}_{b}^{2} \omega_{p b}^{2}\right)
$$


Note that Eq. (35) corresponds to the familiar multi-species Weibel instability (Sec. III. A) in the absence of applied focusing field. On the other hand, in the limit of a strong magnetic field with $\bar{\beta}_{b}^{2} \omega_{p e}^{2} / \omega_{c e}^{2}<<1$, it follows that Eq. (34) can be approximated by

$$
1+\frac{\omega_{p e}^{2}}{\omega_{c e}^{2}}-\frac{\omega_{p e}^{2} \cos ^{2} \theta}{\omega^{2}}-\frac{\omega_{p b}^{2}}{\left(\omega-k_{z} \bar{V}_{b}\right)^{2}}-\frac{\omega_{p i}^{2}}{\left(\omega-k_{z} \bar{V}_{i}\right)^{2}}=\frac{\omega_{p i}^{2} \omega_{p b}^{2}\left(\bar{\beta}_{b}-\bar{\beta}_{i}\right)^{2}}{\left(\omega-k_{z} \bar{V}_{b}\right)^{2}\left(\omega-k_{z} \bar{V}_{i}\right)^{2}} .
$$

Depending on the value of $\cos \theta=k_{z} / k$, the dispersion relation (36) supports solutions ranging from the electrostatic modified two-stream instability between the plasma electrons and the beam ions or the plasma ions, to a streaming instability due to interaction of the ion beam with lower hybrid oscillations [80].

The growth rate of these instabilities have been obtained numerically by solving the full dispersion relation in [80], as well as the approximate dispersion relations in Eqs. (34) and (36). Typical results are illustrated in Figs. 12 and 13. Figure 12 shows the normalized two-stream growth rate $\operatorname{Im} \omega / \omega_{p e}$ plotted as a function of the normalized wavenumber $c k_{z} / \omega_{p e}$ for $c k_{\perp} / \omega_{p e}=20$ and $\omega_{p e} / \omega_{c e}=2$. The dotted curve is the numerical solution of the full electromagnetic dispersion relation [80], and the solid curve is the solution of the (approximate) electrostatic dispersion relation in Eq. (36). Figure 13 shows the normalized growth rate of the multispecies Weibel instability, $\operatorname{Im} \omega / \sqrt{\omega_{p b}^{2}+\omega_{p i}^{2}}$, plotted as a function of $\bar{\beta}_{b} \omega_{p e} / \omega_{c e}$. Note that the growth rate is relatively insensitive to the value of wavenumber $k$ at these very short wavelengths. Figure 13 has been obtained by solving full electromagnetic dispersion relation [80] (dotted line), and by using the approximate dispersion relation in Eq. (36) (solid line) for the choice of system parameters $\quad k_{z}=0, \quad c k_{\perp} / \omega_{p e}=20, \quad \bar{\beta}_{b}=\bar{V}_{b} / c=0.1$, $\omega_{p b} / \omega_{p e}=0.01, \omega_{p i} / \omega_{p b}=1$, and $\hat{n}_{b} / \hat{n}_{i}=0.2$.

In summary, streaming instabilities of intense charged particle beams propagating along a solenidal magnetic field in a background plasma have been studied analytically and numerically [80]. It is found that the growth rates of the multispecies Weibel instability and electrostatic twostream instabilities are strongly affected by the strength of the solenoidal field as measured by the dimensionless parameter $\beta_{b} \omega_{p e} / \omega_{c e}$. 


\section{CONCLUSIONS}

This paper presented a survey of collective processes and beam-plasma interactions affecting heavy ion beam propagation for ion-beam-driven high energy density physics and heavy ion fusion applications. The topics covered in Sec. II included a discussion of the sufficient condition for quiescent (stable) beam propagation over long distances (Sec. II.A); and the electrostatic Harris-type instability (Sec. II.B) and the electromagnetic Weibel-type instability (Sec. II.C) in strongly anisotropic, one-component nonneutral beams. In Sec. III, collective processes associated with the interaction of an intense ion beam pulse with a largevolume, charge-neutralizing background plasma were described. To achieve the high focal spot intensities necessary for high energy density physics and heavy ion fusion applications, compressing the beam longitudinally and transversely in the presence of a dense chargeneutralizing background plasma has many attractive features, particularly because the plasma electrons eliminate (or significantly reduce) the large, defocusing space-charge force of the ion beam pulse. The collective beam-plasma interaction processes summarized in Sec. III included: the multispecies electromagnetic Weibel instability (Sec. III.A); the electrostatic electron-ion two-stream instability (Sec. III.B); dynamic stabilization of the two-stream instability during longitudinal drift compression (Sec. III.C); and the effects of solenoidal magnetic field on collective beam-plasma instabilities (sec. III.D). Operating regimes to minimize the deleterious effects of collective instabilities have been identified.

\section{ACKNOWLEDGMENTS}

This research was supported by the U.S. Department of Energy.

\section{REFERENCES}

[1] B. G. Logan, J. J. Barnard, F. Bieniosek, C. M Celata, R. C. Davidson, A. Friedman, E. Gilson, I. Kaganovich, J. W. Kwan, M. Leitner, A. Molvik, H. Qin, P. Roy, A. Sefkow, P.A. Seidl, E.A. Startsev, S.S. Yu, W.W. Waldron, R.J. Briggs, R.A. Kishek, D.R. Welch and C. Olson, Nucl. Instr. Meth. Phys. Res. A577, 1 (2007).

[2] R. C. Davidson, B. G. Logan, J. J. Barnard et al., Journal de Physique France 133, 731 (2006). 
[3] I. D. Kaganovich, E. A. Startsev, A. B. Sefkow and R. C. Davidson, Phys. Rev. Lett. 99, 235002 (2007).

[4] D. R. Welch, D. V. Rose, C Thoma, A. B. Sefkow, I. D. Kaganovich, P. A. Seidl, S.S.Yu and P. K. Roy, Nucl. Instr. Meth. Phys. Res. A577, 231 (2007).

[5] P. A. Seidl, J. Armijo, D. Baca, F. M. Bieniosek, J. Coleman, E. Henestroza, M. Leitner, B.G. Logan, P. K. Roy, W.L. Waldron, S. S. Yu, A. Friedman, Michel Kireeff-Covo, D. Grote, A. W. Molvik, W. Sharp, J.L. Vay, I. Haber, A. B. Sefkow, E. P. Gilson, P.C. Efthimion, I. Kaganovich, R. C. Davidson, D. Rose and D.R. Welch, Nucl. Instr. Meth. Phys. Res. A577, 215 (2007).

[6] A. B. Sefkow, R. C. Davidson, I. D. Kaganovich, E. P. Gilson, P. K. Roy, S. S. Yu, P. A. Seidl, D. R. Welch, D. V. Rose and J. J. Barnard, Nucl. Instr. Meth. Phys. Res. A577, 289 (2007).

[7] P. K. Roy, S. S. Yu, E. Henestroza, A. Anders, F. M. Bieniosek, J. Coleman, S. Eylon, W. G. Greenway, M. Leitner, B. G. Logan and W. L. Waldron, D. R. Welch and C. Thoma, A. B. Sefkow, E. Gilson, P. C. Efthimion and R. C. Davidson, Phys.Rev. Lett. 95, 234801 (2005).

[8] R. C. Davidson and H. Qin, Physics of Intense Charged Particle Beams in High Energy Accelerators (World Scientific, Singapore, 2001), and references therein.

[9] R. C. Davidson, Physics of Nonneutral Plasmas (World Scientific, Singapore, 2001), and references therein.

[10] M. Reiser, Theory and Design of Charged Particle Beams (John Wiley \& Sons, Inc., New York, 1994).

[11] I.M. Kapchinskij and V.V. Vladimirskij, in Proceedings of the International Conference on High Energy Accelerators and Instrumentation, Geneva, 1959 (CERN, Geneva, 1959), p. 274.

[12] R. L. Gluckstern, in Proceedings of the 1970 Proton Linear Accelerator Conference, Batavia, IL, edited by M. R. Tracy (National Accelerator Laboratory, Batavia, IL, 1971), p. 811.

[13] T.-S.Wang and I. Smith, Part. Accel. 12, 247 (1982).

[14] J. Hofmann, L. J. Laslett, L. Smith, and I. Haber, Part. Accel. 13, 145 (1983).

[15] J. Struckmeier and I. Hofmann, Part. Accel. 39, 219 (1992).

[16] N. Brown and M. Reiser, Phys. Plasmas 2, 965 (1995). 
[17] R.C. Davidson and H. Qin, Phys. Rev. ST Accel. Beams 2, 114401 (1999).

[18] C. Chen and R.C. Davidson, Phys. Rev. E 49, 5679 (1994).

[19] R. L. Gluckstern,W.-H. Cheng, and H.Ye, Phys. Rev. Lett. 75, 2835 (1995).

[20] R.C. Davidson and C. Chen, Part. Accel. 59, 175 (1998).

[21] R.C. Davidson,W.W. Lee, and P. Stoltz, Phys. Plasmas 5, 279 (1998).

[22] C. Chen, R. Pakter, and R.C. Davidson, Phys. Rev. Lett. 79, 225 (1997).

[23] R C. Davidson, Phys. Rev. Lett. 81, 991 (1998).

[24] R.C. Davidson, Phys. Plasmas 5, 3459 (1998).

[25] C. S. Gardner, Phys. Fluids 6, 839 (1963).

[26] P. Stoltz, R.C. Davidson, andW.W. Lee, Phys. Plasmas 6,298 (1999).

[27] W.W. Lee, Q. Qian, and R.C. Davidson, Phys. Lett. A 230, 347 (1997).

[28] Q. Qian,W.W. Lee, and R. C. Davidson, Phys. Plasmas 4, 1915 (1997).

[29] R.C. Davidson and H. Qin, Phys. Rev. ST Accel. Beams 4, 104401 (2001).

[30] S. I. Tzenov and R. C. Davidson, Phys. Rev. ST Accel. Beams 5, 021001 (2002).

[31] R.C. Davidson, H. Qin, and P. J. Channell, Phys. Rev. ST Accel. Beams 2, 074401 (1999);

3, 029901 (2000).

[32] E.G. Harris, Phys. Rev. Lett. 2, 34 (1959).

[33] E. A. Startsev, R.C. Davidson, and H. Qin, Phys. Plasmas 9, 3138 (2002).

[34] E. A. Startsev, R.C. Davidson, and H. Qin, Laser Part. Beams 20, 585 (2002).

[35] E. A. Startsev, R.C. Davidson, and H. Qin, Phys. Rev. ST Accel. Beams 6, 084401 (2003).

[36] E. A. Startsev, R.C. Davidson and H. Qin, Nucl. Instr. Meth. Phys. Res. A 544, 125 (2005).

[37] E.A. Startsev, R.C. Davidson and H. Qin, Phys. Rev. ST Accel. Beams 8, 124201 (2005).

[38] H. Qin, R. C. Davidson and E. A. Startsev, Phys. Rev. ST Accel. Beams 10, 064201 (2007).

[39] E.A. Startsev, R. C. Davidson and H Qin, Phys. Plasmas 14, 056705 (2007).

[40] R. A. Kishek, P.G. O’Shea, and M. Reiser, Phys. Rev. Lett. 85, 4514 (2000).

[41] J. Haber, A. Friedman, D. P. Grote, S.M. Lund, and R. A. Kishek, Phys. Plasmas 6, 2254 (1999).

[42] A. Friedman, D. P. Grote, and I. Haber, Phys. Fluids B 4, 2203 (1992).

[43] E. S.Weibel, Phys. Rev. Lett. 2, 83 (1959).

[44] E. A. Startsev and R.C. Davidson, Phys. Plasmas 10, 4829 (2003).

[45] W.W. Lee, R.C. Davidson, E.A. Startsev and H. Qin, Nucl. Instr. Meth. Phys. Res. A 544, 
353 (2005).

[46] R.C. Davidson, D. A. Hammer, I. Haber, and C. E. Wagner, Phys. Fluids 15, 317 (1972).

[47] C. A. Kapetanakos, Appl. Phys. Lett. 25, 484 (1974).

[48] M. Honda, J. Meyer-ter-Vehn, and A. Pukhov, Phys. Rev. Lett. 85, 2128 (2000).

[49] V. K. Neil and A.M. Sessler, Rev. Sci. Instrum. 36, 429 (1965).

[50] E. P. Lee, Part. Accel. 37, 307 (1992).

[51] R.C. Davidson, H. Qin, and G. Shvets, Phys. Rev. ST Accel. Beams 6, 104402 (2003).

[52] E. P. Lee, Phys. Fluids 21, 1327 (1978).

[53] E. J. Lauer, R. J. Briggs, T. J. Fesendon, R. E. Hester, and E. P. Lee, Phys. Fluids 21, 1344 (1978).

[54] M. N. Rosenbluth, Phys. Fluids 3, 932 (1960).

[55] H. S. Uhm and M. Lampe, Phys. Fluids 23, 1574 (1980).

[56] H. S. Uhm and M. Lampe, Phys. Fluids 24, 1553 (1981).

[57] R. F. Fernsler, S. P. Slinker, M. Lampe, and R. F. Hubbard, Phys. Plasmas 2, 4338 (1995), and references therein.

[58] H. S. Uhm and R. C. Davidson, Phys. Rev. ST Accel. Beams 6, 034204 (2003).

[59] E. Keil. and B. Zotter, Report No. CERN-ISR-TH-71-58, 1971.

[60] D.G. Koshkarev and P. R. Zenkevich, Part. Accel. 3, 1 (1972).

[61] L. J. Laslett, A.M. Sessler, and D. Moehl, Nucl. Instrum. Methods 121, 517 (1974).

[62] D. Neuffer, B. Colton, D. Fitzgerald, T. Hardek, R. Hutson, R. Macek, M. Plum, H.

Thiessen, and T. S. Wang, Nucl. Instrum. Methods Phys. Res., A 321, 1 (1992).

[63] R. J. Macek et al., in Proceedings of the 2001 Particle Accelerator Conference, Chicago (IEEE, Piscataway, NJ, 2001), Vol. 1, p. 688.

[64] R. C. Davidson, H. Qin, and T.-S. Wang, Phys. Lett. A 252, 213 (1999).

[65] R.C. Davidson, H. Qin, P. H. Stoltz, and T.-S.Wang, Phys. Rev. ST Accel. Beams 2 , 054401 (1999).

[66] R.C. Davidson and H. Qin, Phys. Lett. A 270, 177 (2000).

[67] H. Qin, R.C. Davidson, and W.W. Lee, Phys. Rev. ST Accel. Beams 3, 084401 (2000); 3, 109901 (2000).

[68] H. Qin, R. C. Davidson, E. A. Startsev, and W.W. Lee, Laser Part. Beams 21, 21 (2003). 
[69] T.-S. Wang, P. J. Channell, R. J. Macek, and R. C. Davidson, Phys. Rev. ST Accel. Beams 6, 014204 (2003).

[70] H. Qin, Phys. Plasmas 10, 2078 (2003).

[71] H. Qin, E. A. Startsev, and R. C. Davidson, Phys. Rev. ST Accel. Beams 6, 014401 (2003).

[72] I. D. Kaganovich, A. B. Sefkow, E.A. Startsev, R. C. Davidson and D. R. Welch, Nucl. Instr. Meth. Phys. Res. A577, 93 (2007).

[73] I. D. Kaganovich, E. A. Startsev, R. C. Davidson and D. R. Welch, Nucl. Instr. Meth. Phys. Res. A544, 383 (2005).

[74] I. D. Kaganovich, E. A. Startsev, and R.C. Davidson, Laser Part. Beams 20, 497 (2002).

[75] I. D. Kaganovich, G. Shvets, E. Startsev, and R.C. Davidson, Phys. Plasmas 8, 4180 (2001).

[76] D.V. Rose, P. F. Ottinger, D. R. Welch, B.V. Oliver, and C. L. Olson, Phys. Plasmas 6, 4094 (1999).

[77] D. R. Welch, D.V. Rose, B.V. Oliver, T.C. Genoni, C. L. Olson, and S. S. Yu, Phys. Plasmas 9, 2344 (2002).

[78] R. C. Davidson, I. D. Kaganovich, H. Qin, E. A. Startsev, D. R. Welch, D. V. Rose and H. S. Uhm, Phys. Rev. ST Accel. Beams 7, 114801 (2004).

[79] R. C. Davidson, M. Dorf, I. D. Kaganovich, H. Qin, A. B. Sefkow and E. A. Startsev, D. R. Welch, D. D. Rose and S. M. Lund, Nucl. Instr. Meth. Phys. Res. A577, 70 (2007).

[80] E. A. Startsev, R.C. Davidson and M. Dorf, Phys. Plasmas 15, 062107 (2008).

[81] E. A. Startsev and R. C. Davidson, Nucl. Instr. Meth. Phys. Res. A577, 79 (2007).

[82] E. A. Startsev and R. C. Davidson, Phys. Plasmas 13, 062108 (2006); D. V. Rose, T. C. Genoni, D. R. Welch, E. A. Startsev and R. C. Davidson, Phys. Rev. ST Accel. Beams 10, 034203 (2007)

[83] See also Chap. 4 and Sec. 8.6 of Ref. [8].

[84] R.C. Davidson, I. Kaganovich, and E. A. Startsev, "Weibel and Two-Stream Instabilities for Intense Charged Particle Beam Propagation Through Neutralizinig Background Plasma”, Princeton Plasma Physics Laboratory Report No. PPPL-3940, 2004.

[85] See, for example, Sec. 9.5 of Ref. [9]. 


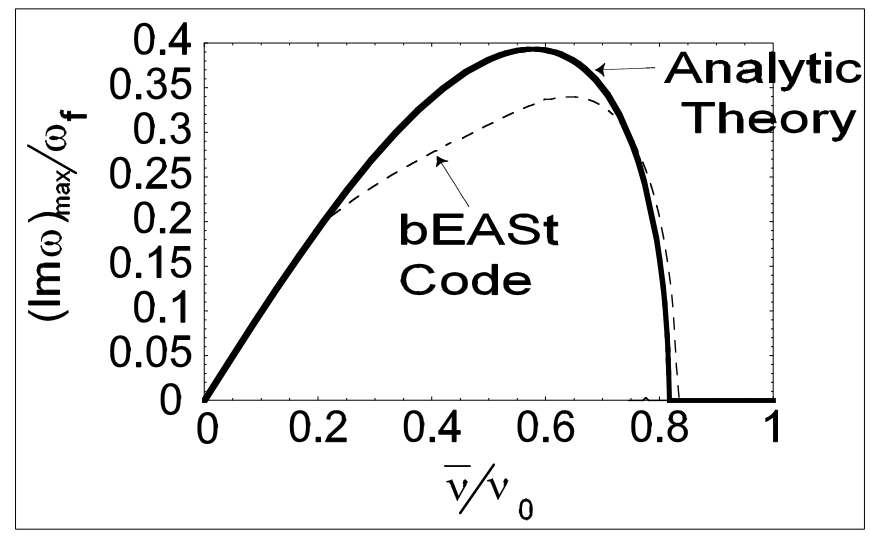

Fig. 1. Plots of normalized growth rate $(\operatorname{Im} \omega)_{\max } / \omega_{f}$ versus normalized tune $\bar{v} / v_{0}$ for $T_{\| b} / T_{\perp b}=0$ and azimuthal mode number $m=1$ (dashed curve) [39]. Results have been obtained using the eigenmode code bEASt $[37,39]$. The solid curve corresponds to the simple theoretical estimate in [37].

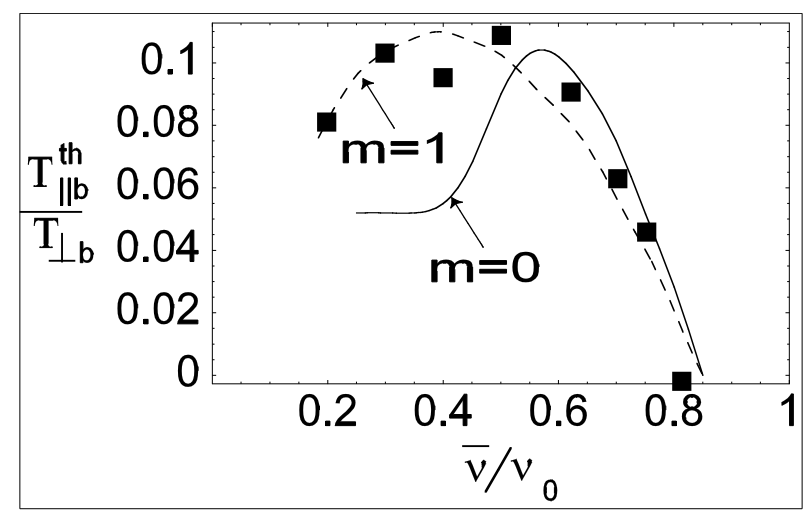

Fig. 2. The threshold value of longitudinal beam temperature $T_{\| b}^{t h}$ for onset of the Harris instability normalized to the transverse temperature $T_{\perp b}$ is plotted versus the normalized tune depression $\bar{v} / v_{0}$ for two values of the azimuthal mode number, $m=0$ (solid line) and $m=1$ (dashed line) [39]. The squares correspond to the effective longitudinal beam temperature $T_{\| b} \equiv m_{b}\left\langle v_{\|}^{2}\right\rangle$ obtained from simulations using the nonlinear $\delta f$ code after the instability saturates. 


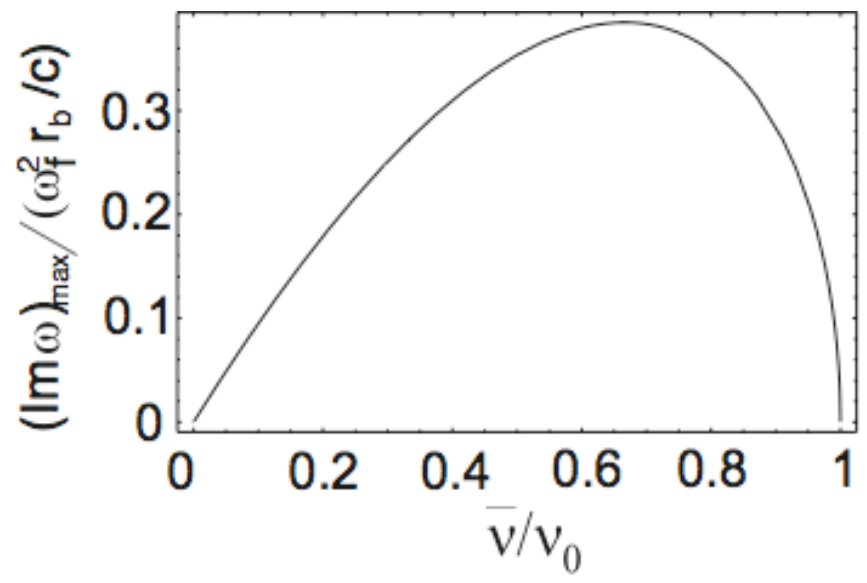

Fig. 3. Plot of the normalized maximum growth rate $(\operatorname{Im} \omega)_{\max } /\left(\omega_{f}^{2} r_{b} / c\right)$ of the Weibel instability versus normalized tune $\bar{v} / v_{0}$ obtained from Eq. (8).

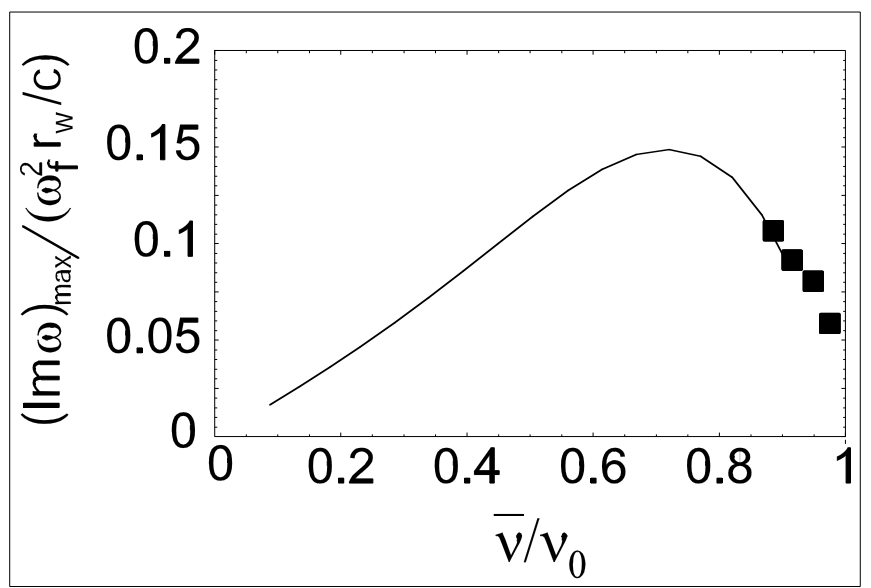

Fig. 4. Plot of normalized maximum growth rate $(\operatorname{Im} \omega)_{\max } /\left(\omega_{f}^{2} r_{b} / c\right)$ of the Weibel instability versus normalized tune $\bar{v} / v_{0}$ for $T_{\| b} / T_{\perp b}=0$ and wall radius $r_{w}=3 r_{b}$ [39]. 


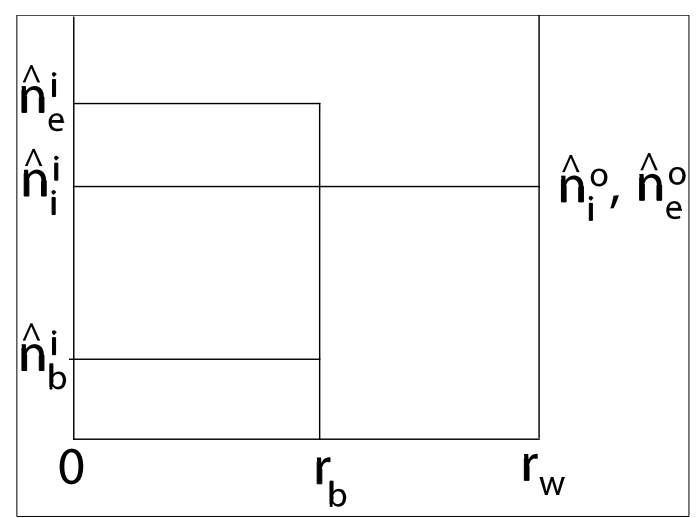

Fig. 5. Schematics of the density profiles of the beam ions $\left(\hat{n}_{b}^{i}\right)$ and the plasma ions and electrons inside $\left(\hat{n}_{i}^{i}\right.$ and $\left.\hat{n}_{e}^{i}\right)$ and outside $\left(\hat{n}_{i}^{o}\right.$ and $\left.\hat{n}_{e}^{o}\right)$ the beam.

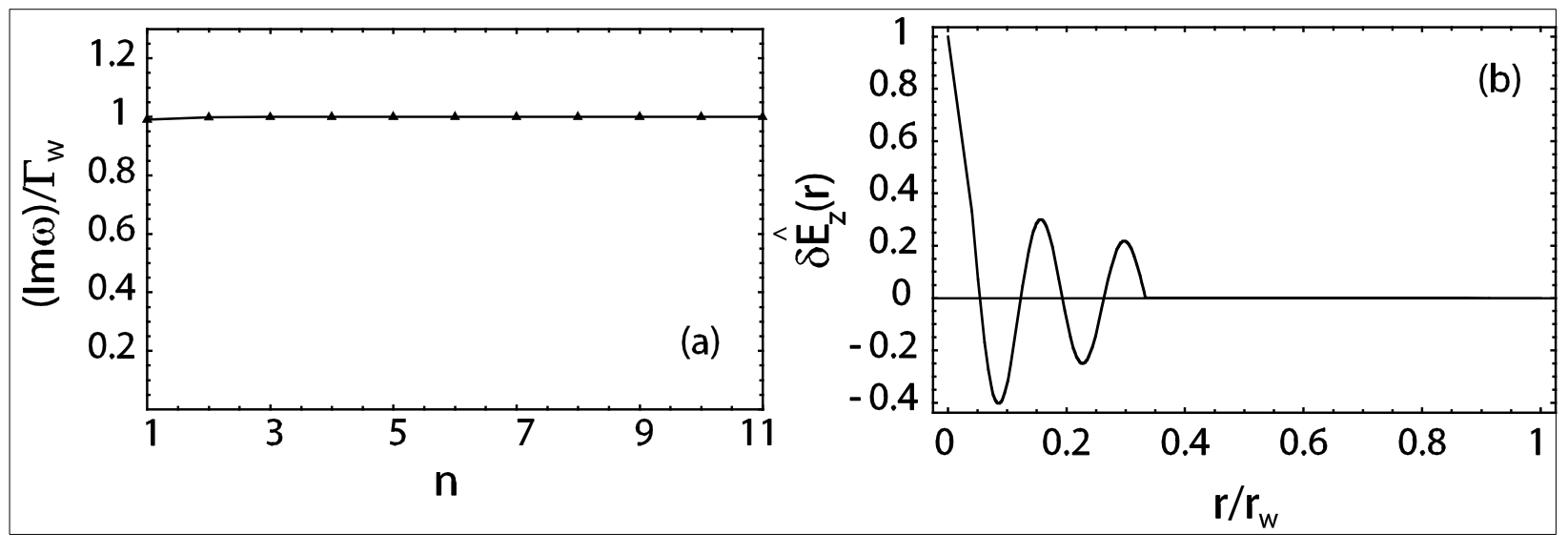

Fig. 6. Plots of (a) Weibel instability growth rate $\operatorname{Im} \omega / \Gamma_{W}$ versus radial mode number $n$, and (b) eigenfunction $\delta \hat{E}_{z}(r)$ versus $r / r_{w}$ for $n=5$ [79]. System parameters are $r_{b}=r_{w} / 3, \beta_{b}=0.2$, $\beta_{e}=0.1, \hat{n}_{i}^{i}=\hat{n}_{e}^{i} / 2=\hat{n}_{b}^{i}=\hat{n}_{e}^{o}=\hat{n}_{i}^{o}, \Omega_{p}^{i} r_{b} / c=1 / 3$. 

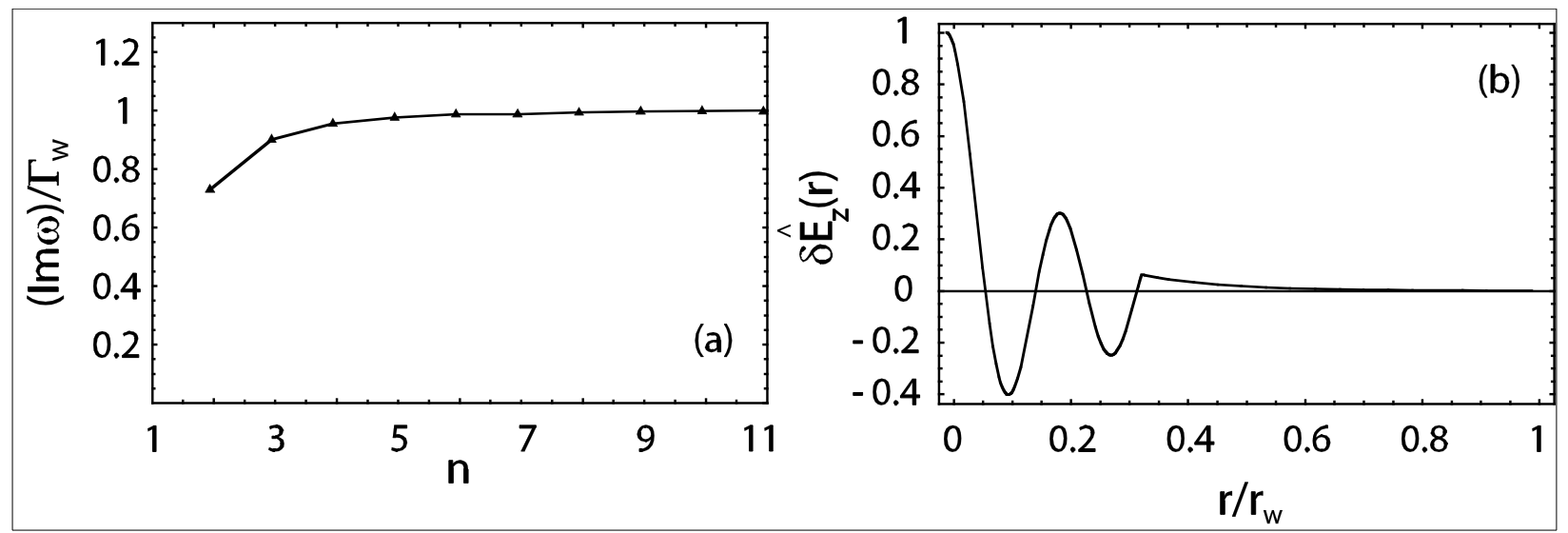

Fig. 7. Plots of (a) Weibel instability growth rate $\operatorname{Im} \omega / \Gamma_{W}$ versus radial mode number $n$, and (b) eigenfunction $\delta \hat{E}_{z}(r)$ versus $r / r_{w}$ for $n=5$ [79]. System parameters are $r_{b}=r_{w} / 3, \beta_{b}=0.2$, $\beta_{e}=0.1, \hat{n}_{i}^{i}=\hat{n}_{e}^{i} / 2=\hat{n}_{b}^{i}=\hat{n}_{e}^{o}=\hat{n}_{i}^{o}, \Omega_{p}^{i} r_{b} / c=3$.
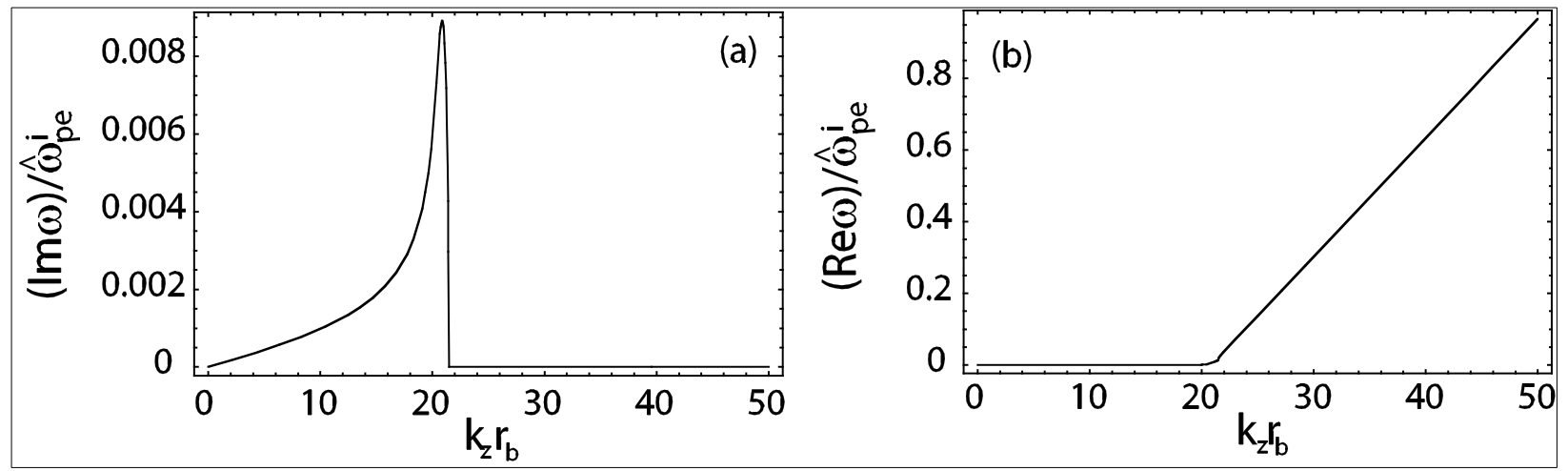

Fig. 8. Plots of (a) $(\operatorname{Im} \omega) / \hat{\omega}_{p e}^{i}$ and (b) $(\operatorname{Re} \omega) / \hat{\omega}_{p e}^{i}$ versus $k_{z} r_{b}$ calculated from the two-stream dispersion relation (22) for $r_{b}=r_{w} / 3, \beta_{b}=0.2, \beta_{e}=0.1$, and $\hat{\omega}_{p e}^{i} r_{b} / c=3$ in the absence of plasma outside the beam plasma channel $[78,84]$. 


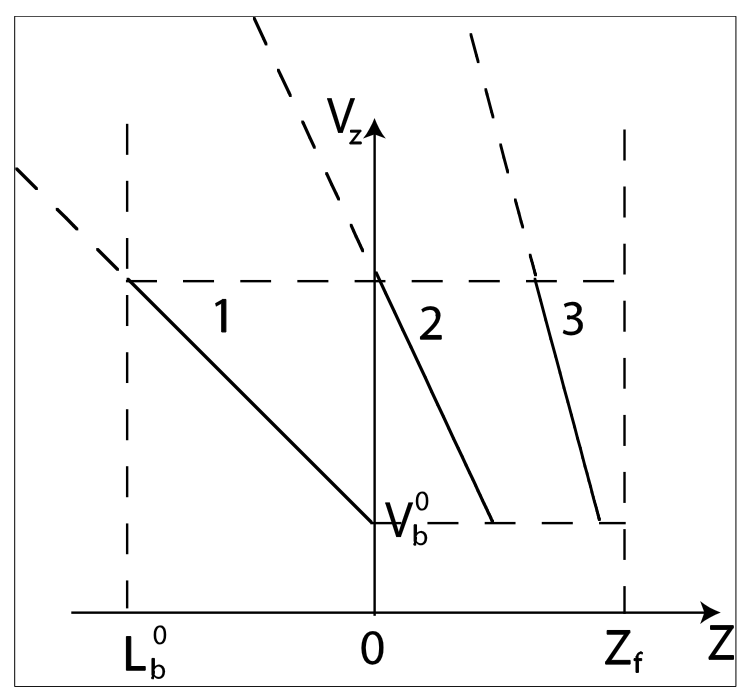

Fig. 9. Plot of ion beam phase space at different times during the compression. Line 1 corresponds to $\mathrm{t}=0$.

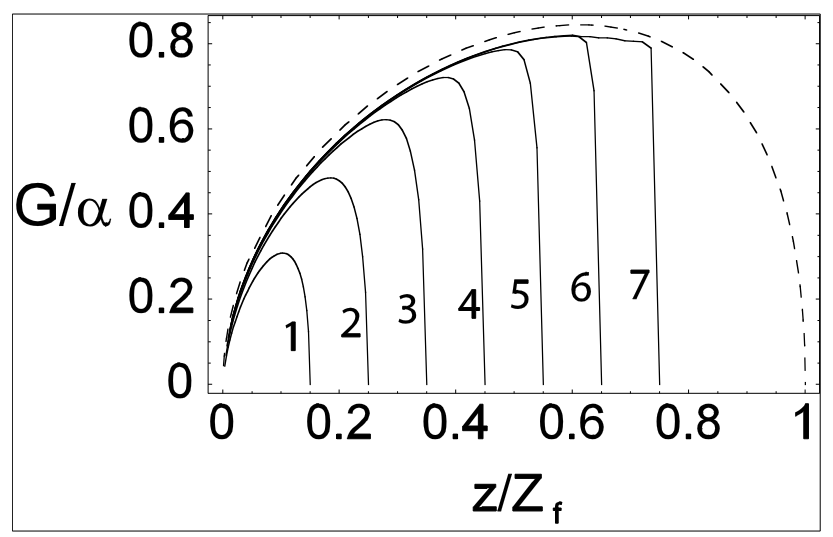

Fig. 10. The normalized instability gain function $G(z, t) / \alpha$ is plotted as a function of distance $z / Z_{f}$ at different times $t / T_{f}=0.15$ (1), 0.25 (2), 0.35 (3), 0.45 (4), 0.55 (5), 0.65 (6), and 0.75 (7) obtained numerically (solid curve) and compared with the analytical estimate (dashed curve). 


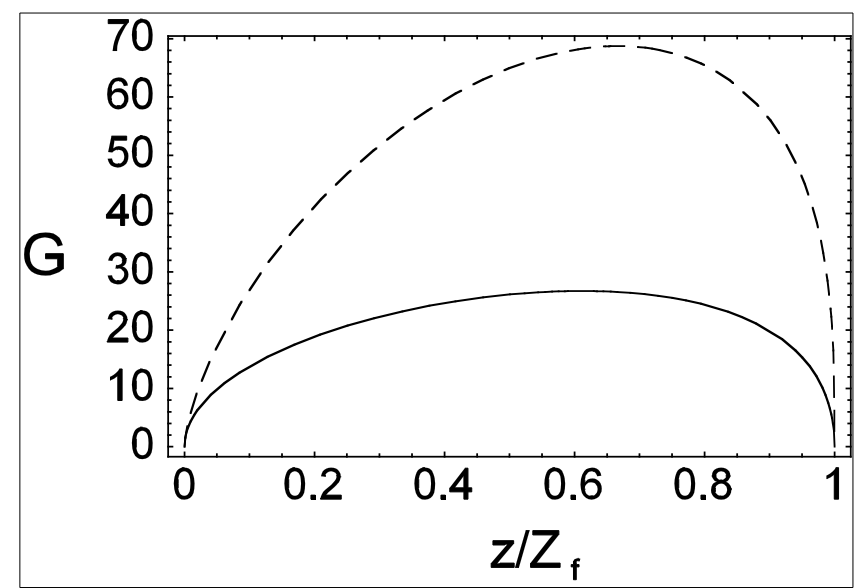

Fig. 11. Comparison of the instability gain as a function of $z / Z_{f}$ for a beam with velocity tilt (solid curve) and without velocity tilt (dashed curve) for $\delta \equiv Z_{b} n_{b}^{0} / \hat{n}_{i}=10^{-3}$ and $\alpha^{2}=\left(\omega_{p b} T_{f}\right)^{2}=1000$

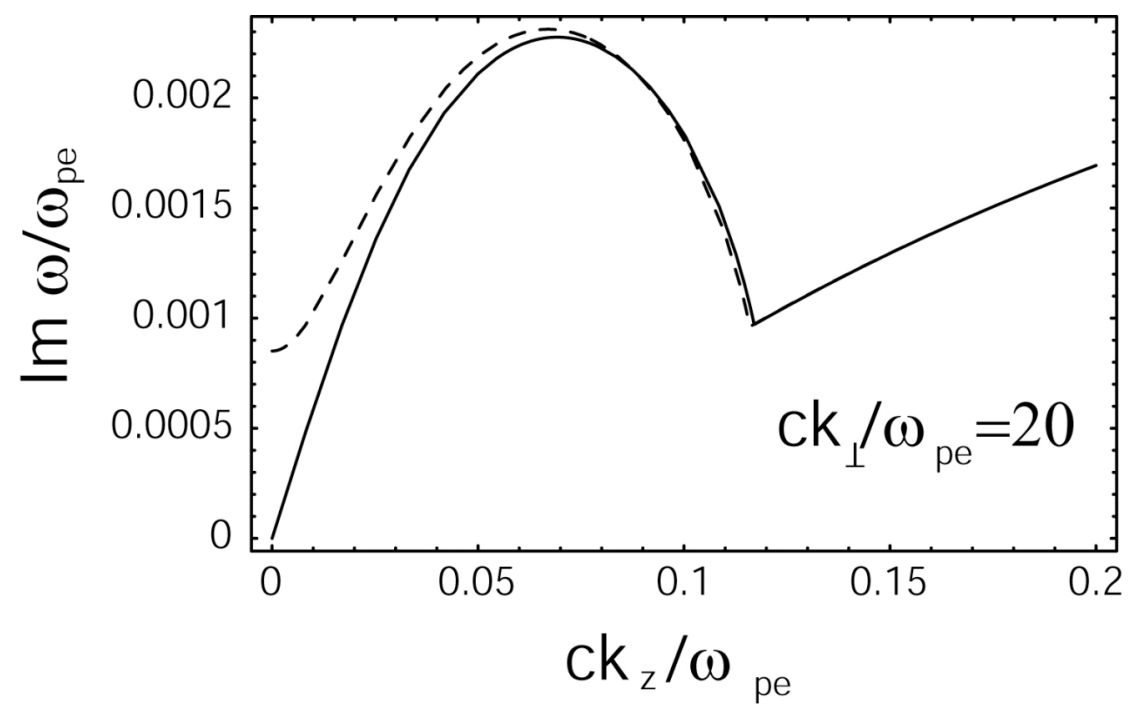

Fig. 12. Normalized two-stream growth rate $\operatorname{Im} \omega / \omega_{p e}$ plotted as a function of normalized wavenumber $c k_{z} / \omega_{p e}$ for $c k_{\perp} / \omega_{p e}=20$ and $\bar{\beta}_{b}=\bar{V}_{b} / c=0.1, \omega_{p b} / \omega_{p e}=0.01, \omega_{p i} / \omega_{p b}=1$, $\omega_{p e} / \omega_{c e}=2, \bar{\beta}_{b} \omega_{p e} / \omega_{c e}=0.2$, and $\hat{n}_{b} / \hat{n}_{i}=0.2$. The dotted line is the solution of the full electromagnetic dispersion relation in [80], and the solid line is the solution of the approximate electrostatic dispersion relation in Eq. (36). 


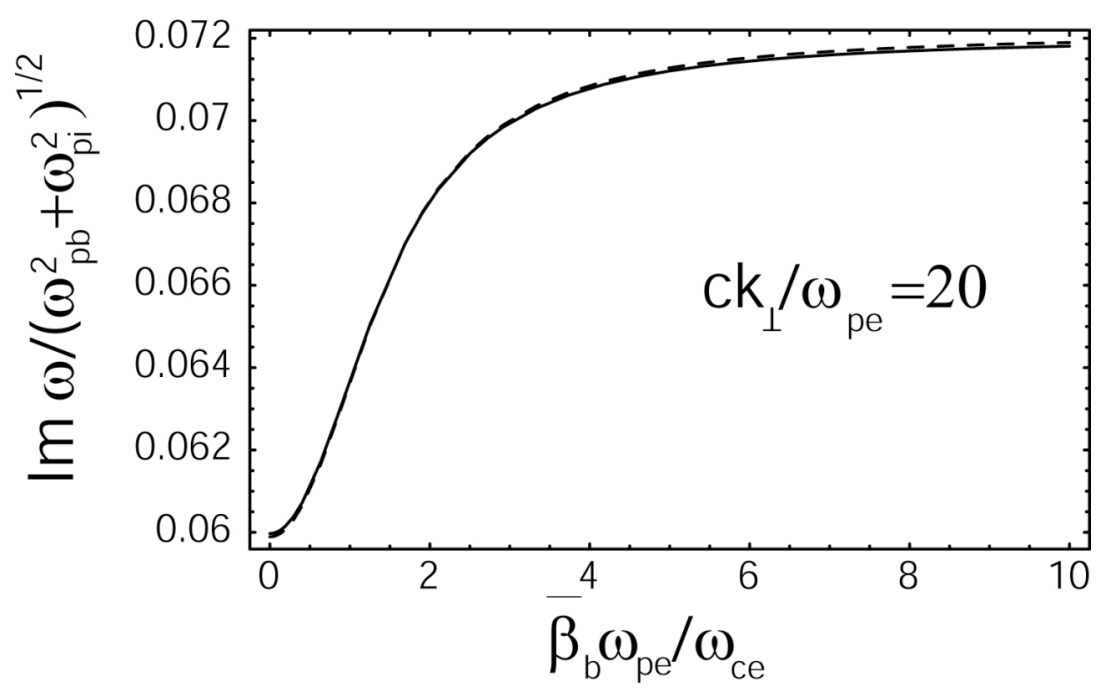

Fig. 13. Normalized growth rate of the Weibel instability $\operatorname{Im} \omega / \sqrt{\omega_{p b}^{2}+\omega_{p i}^{2}}$ plotted as a function of $\bar{\beta}_{b} \omega_{p e} / \omega_{c e}$ for $k_{z}=0, c k_{\perp} / \omega_{p e}=20, \bar{\beta}_{b}=\bar{V}_{b} / c=0.1, \omega_{p b} / \omega_{p e}=0.01$, and $\hat{n}_{b} / \hat{n}_{i}=0.2$. The dotted line is the solution of the full electromagnetic dispersion relation in [80], and the solid line is obtained from the approximate dispersion relation in Eq. (34). 